This item was submitted to Loughborough's Research Repository by the author.

Items in Figshare are protected by copyright, with all rights reserved, unless otherwise indicated.

\title{
Predicting the critical heat flux in pool boiling based on hydrodynamic instability induced irreversible hot spots
}

\section{PLEASE CITE THE PUBLISHED VERSION}

https://doi.org/10.1016/j.ijmultiphaseflow.2018.02.021

\section{PUBLISHER}

(c) Elsevier

VERSION

AM (Accepted Manuscript)

\section{PUBLISHER STATEMENT}

This work is made available according to the conditions of the Creative Commons Attribution-NonCommercialNoDerivatives 4.0 International (CC BY-NC-ND 4.0) licence. Full details of this licence are available at: https://creativecommons.org/licenses/by-nc-nd/4.0/

\section{LICENCE}

CC BY-NC-ND 4.0

\section{REPOSITORY RECORD}

Zhao, Huayong, and Andrew M. Williams. 2018. "Predicting the Critical Heat Flux in Pool Boiling Based on Hydrodynamic Instability Induced Irreversible Hot Spots". Loughborough University. https://hdl.handle.net/2134/32135. 


\section{Predicting the critical heat flux in pool boiling based on hydrodynamic instability induced irreversible hot spots}

Abbreviated title: Critical heat flux in pool boiling

Huayong Zhao ${ }^{1^{*}}$, Andrew Williams ${ }^{2}$

1. Wolfson School of Mechanical, Electrical and Manufacturing Engineering Loughborough University, Leicestershire, LE11 3TU, United Kingdom

2. Department of Mechanical Engineering, Faculty of Science and Engineering, University of Chester, Chester, CH1 4BJ, United Kingdom

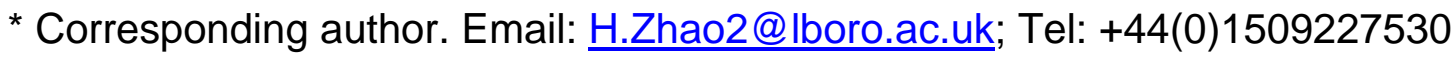

Email of the co-author: Andrew.Williams@Chester.ac.uk

\section{Abstract}

A new model, based on the experimental observation reported in the literature that CHF is triggered by the Irreversible Hot Spots (IHS), has been developed to predict the Critical Heat Flux (CHF) in pool boiling. The developed Irreversible Hot Spot (IHS) model can predict the CHF when boiling methanol on small flat surfaces and long horizontal cylinders of different sizes to within $5 \%$ uncertainty. It can also predict the effect of changing wettability (i.e. contact angle) on CHF to within $10 \%$ uncertainty for both hydrophilic and hydrophobic surfaces. In addition, a linear empirical correlation has been developed to model the bubble growth rate as a function of the system pressure. The IHS model with this linear bubble growth coefficient correlation can predict the $\mathrm{CHF}$ when boiling water on both flat surfaces and long horizontal cylinders to within $5 \%$ uncertainty up to 10 
bar system pressure, and the CHF when boiling methanol on a flat surface to within $10 \%$ uncertainty up to 5 bar. The predicted detailed bubble grow and merge process from various sub-models are also in good agreement with the experimental results reported in the literature.

\section{Keywords (max. 6)}

Critical heat flux, pool boiling, hydrodynamic instability, contact angle, pressure

\section{Introduction}

Boiling has been widely applied in many important industrial applications, such as in the nuclear industry and high power electronic systems, as an efficient way to manage the excessive thermal load. The ability of a boiling system to dissipate heat at different surface temperatures has been extensively investigated since the early 1970s and is often represented by the well-known Nukiyama curve (i.e. the boiling curve). The boiling curve suggests that there is a maximum heat flux (i.e. Critical Heat Flux - CHF) condition at the end of the nucleate boiling regime. When the imposed heat flux is larger than the CHF, the surface temperature will increase significantly to the so-called burnout temperature, which is typically well above the softening point or even the melting point of the metal surface, often causing system failure. Therefore, significant efforts have gone into understanding and predicting $\mathrm{CHF}$ through both experiments and simulations. The recent advancements in measurement and imaging techniques have made it possible to visualise and quantify the conditions near CHF directly. These experimental results show that the process behind the initiation of the CHF is contradictory to most of the postulated 
physical processes behind existing models. Furthermore, these results provide clues and insights for the development of new CHF models based on more rigorous physics. This paper firstly reviews the existing models and discusses their limitations and contradictions to the observed physical process. It then presents a new model which is better capable of predicting the CHF conditions.

\section{Review of existing models and their limitations}

The existing models used to predict the CHF in pool boiling can be broadly grouped into four different types of models: (i) the hydrodynamic instability models, (ii) the hydrodynamic force imbalance model, (iii) the macrolayer dryout models, and (iv) the dry spots models. Different types of models are derived based on different postulated CHF initiation mechanisms. The formulae, the key underlying assumptions, and the limitations of these models are reviewed in turn.

\section{(i) The hydrodynamic instability models}

The hydrodynamic instability models have been the most popular models since their first appearance in 1950 by Kuteladze (Kutateladze, 1950) who derived it through non-dimensional analysis. The model was then further developed into many widely adopted analytical models, such as Zuber's model (Zuber, 1959), Lienhard and Dhir's model (Dhir and Lienhard, 1973) and many other semiempirical models which aim to correct the Zuber's model by multiplying it with extra terms obtained through curve fitting to experimental data (e.g. (El-Genk, Mohamed S.; Guo, 1993)(Brusstar, Matthew J.; Merte, 1994)). Yagov (Yagov, 2014) provided a comprehensive review of this group of models and concluded 
that some of the critical assumptions could not be justified or were contradictory to the experimental observations.

The detailed assumptions behind this group of models can be found in the literature (Zuber, 1959; Carey, 2008; Liang and Mudawar, 2017). The key controversial assumptions are presented here.

1. The vapour columns are assumed to be cylindrical and distributed based on the two-dimensional wave patterns predicted by the Rayleigh-Taylor (RT) instability. Zuber (Zuber, 1959) used the critical wavelength $\left(\lambda_{C, R T}\right)$ and the most dangerous wavelength $\left(\lambda_{D, R T}=\sqrt{3} \lambda_{C, R T}\right)$ to calculate the upper and lower limit in CHF. Lienhard and Dhir (Dhir and Lienhard, 1973) used the most dangerous wavelength $\left(\lambda_{D, R T}\right)$. The radius of the vapour column $\left(R_{v}\right)$ in both models are assumed to be equal to one quarter of the unstable wavelength.

2. The Kelvin-Helmholtz $(\mathrm{KH})$ instability wavelength $\left(\lambda_{K H}\right)$ imposed on the columns in Zuber's model is assumed to be equal to the PlateauRayleigh instability wavelength for circular jets $\left(\lambda_{K H, Z}=2 \pi R_{v}\right) . \lambda_{K H}$ in the Lienhard and Dhir's model is assumed to be equal to the most dangerous wavelength predicted by the RT instability theory $\left(\lambda_{K H, L}=\right.$ $\left.\lambda_{D, R T}=\sqrt{3} \lambda_{c, R T}\right)$

The $\mathrm{KH}$ instability analysis for a vertical interface between the liquid phase and vapour phase suggests the critical velocity difference $\left(u_{c}\right)$ between the liquid phase and the vapour phase can be calculated by (Carey, 2008): 
$u_{c}=\left|\bar{u}_{l}-\bar{u}_{v}\right|=\left[\frac{2 \pi \sigma\left(\rho_{l}+\rho_{v}\right)}{\rho_{l} \rho_{v} \lambda_{K H}}\right]^{1 / 2}$

where $\bar{u}_{l}$ and $\rho_{l}$ are the average velocity and the density of the liquid phase flow; $\bar{u}_{v}$ and $\rho_{v}$ is the average velocity and the density of the vapour phase flow; $\sigma$ is the surface tension; and $\lambda_{K H}$ is the critical wavelength to induce the $\mathrm{KH}$ instability.

Since $\rho_{l} \gg \rho_{v}$, to satisfy the continuity Equation, $u_{c} \cong u_{v} \cong\left[\frac{2 \pi \sigma}{\rho_{v} \lambda_{K H}}\right]^{1 / 2}$

According to assumptions 1 and 2,

$q_{C H F}^{\prime \prime}=\rho_{v} u_{v} h_{l v}\left(\frac{A_{v}}{A_{s}}\right)=\rho_{v} h_{l v}\left(\frac{\pi}{16}\right)\left[\frac{2 \pi \sigma}{\rho_{v} \lambda_{K H}}\right]^{1 / 2}$

where $A_{s}$ is the surface area; $A_{v}$ is the vapour column area; and $h_{l v}$ is the latent heat.

The $\mathrm{RH}$ instability analysis ( $\mathrm{KH}$ instability for a horizontal surface with negligible interface velocity: $\bar{u}_{l}=\bar{u}_{v}=0$ ) suggests:

$\lambda_{D, R T}=2 \pi\left[\frac{3 \sigma}{\left(\rho_{l}-\rho_{v}\right) g}\right]^{1 / 2}$

The arithmetic average of the upper limit (i.e. $\lambda_{K H}=\lambda_{C, R T}$ ) and the lower limit (i.e. $\lambda_{K H}=\lambda_{D, R T}$ ) can be calculated by Eq. (4a) (Zuber, 1959):

$q_{C H F, Z}^{\prime \prime}=\frac{\left(q_{C H F, Z, u p p e r}^{\prime \prime}+q_{C H F, Z, \text { lower }}^{\prime \prime}\right)}{2}=0.138 \rho_{v} h_{l v}\left[\frac{\sigma\left(\rho_{l}-\rho_{v}\right) g}{\rho_{v}^{2}}\right]^{1 / 4}$

Zuber also introduced a convenient way to get the average value, which is using $\lambda_{C, R T}$ as the instability wavelength but scale down the coefficient (Zuber, 
1959). The result is Eq. (4b) and is widely adopted as the Zuber's model to calculate the $\mathrm{CHF}$ in literature.

$$
q_{C H F, Z}^{\prime \prime}=0.131 \rho_{v} h_{l v}\left[\frac{\sigma\left(\rho_{l}-\rho_{v}\right) g}{\rho_{v}^{2}}\right]^{1 / 4}
$$

Lienhard and Dhir's model (Dhir and Lienhard, 1973) can be written as a function of Zuber's model such that: $q_{C H F, L D}^{\prime \prime}=1.14 q_{C H F, Z}^{\prime \prime}$.

Both analytical hydrodynamic instability models are susceptible to the choice of the $\mathrm{KH}$ wavelength $\left(q_{C H F}{ }^{\prime \prime} \propto \lambda_{K H}^{-1 / 2}\right)$ and the area ratio between the columns and surface $\left(q_{C H F}{ }^{\prime \prime} \propto A_{v} / A_{s}\right)$. Both models used the instability wavelength from the $\mathrm{RT}$ instability as the $\mathrm{KH}$ instability wavelength. However, the RT instability is only a special case of the $\mathrm{KH}$ instability where a high-density fluid is on top of a low-density fluid with negligible interfacial velocity, which is quite different from the actual flow conditions in boiling. The sizes and the separation distances of vapour columns used in these two models also cannot be justified. As indicated by Yagov (Yagov, 2014), the experimental results reported by various researchers contradict the sizes and the separation distances of the vapour columns used in these two models. Many experimental visualizations (Ahn and Kim, 2012; Chu, No and Song, 2013; Chu et al., 2014) also clearly show that the vapour generated under high heat flux conditions (including the CHF condition) is encapsulated into large coalesced bubbles which repeatedly form and detach from the surface. In addition, the bubble sizes were shown to reach diameter much larger than $\lambda_{D, R T} / 4$. Therefore, the steady-state condition behind the hydrodynamic instability models is not an accurate representation of the actual physical process. 
Despite these difficulties, the analytical models developed by Zuber, and, Lienhard and Dhir can predict the CHF in saturated pool boiling on smooth horizontal surfaces to within $20 \%$ accuracy, which is about the level of scattering in data of this type (Carey, 2008). The most accurate models used to predict CHF conditions are mostly semi-empirical correlations modified from the Zuber's model (Fang and Dong, 2016). These models usually incorporate an empirically fitted function of the contact angle which is overlooked in both Zuber's model and the Lienhard and Dhir's model. This suggests that the existing analytical hydrodynamic instability model is likely to be an incomplete model which should be revised to represent the experimental observations more accurately.

\section{(ii) Hydrodynamic forces imbalance model}

Kandlikar developed a model based on the hydrodynamic behaviour of the interface of a single detached bubble to predict the $q_{C H F}^{\prime \prime}$ (Kandlikar, 2001). Kandlikar considered the force balance for a single large bubble, as shown in Fig. 1. He hypothesises that the CHF occurs when the repulsive force coming from the liquid evaporation on the interface surpasses the surface tension force and gravitational forces along the evaporation direction so that the bubble will be stretched sideways to merge with the adjacent bubble to form a vapour blanket, which in turn covers part or the whole heating surface to trigger the CHF. The surface tension forces at the top and the bottom of the bubbles are calculated based on the contact angle of the droplet. The repulsive force is calculated by treating the bubble interface to be cylindrical. The effect of surface inclination is taken into account when decomposing the gravity force along the 
direction parallel to the heating surface. Kandlikar further assumes the average bubble size can be taken as $\lambda_{C, R T} / 2$. The $q_{C H F, K}^{\prime \prime}$ can be calculated as (Kandlikar, 2001):

$q_{C H F, K}^{\prime \prime}=h_{l v} \cdot \rho_{v} \cdot\left[\frac{\sigma g\left(\rho_{l}-\rho_{v}\right)}{\rho_{v}^{2}}\right]^{1 / 4} \cdot \frac{1+\cos \beta}{16} \cdot\left[\frac{2}{\pi}+\frac{\pi}{4}(1+\cos \beta) \cos \phi\right]^{1 / 2}$

where $\beta$ is the dynamic receding contact angle and $\phi$ is the surface inclination angle ( $\phi=0$ for horizontal surfaces).

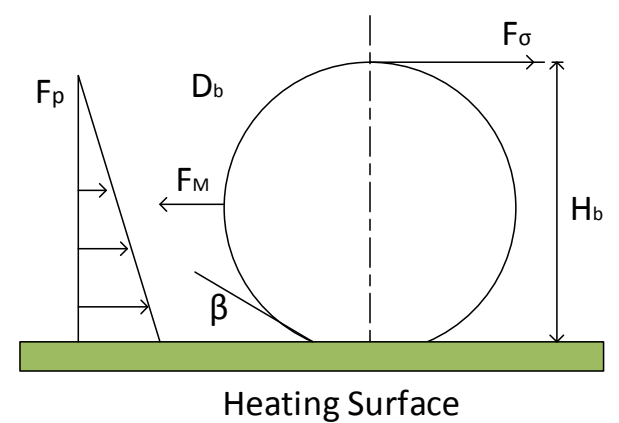

Fig. 1 Force-imbalance on bubbles in CHF in Kandlikar's model

Comparison with the experimental data (Kandlikar, 2001; Fang and Dong, 2016) indicates the Kandlikar's model can predict the $q_{C H F}^{\prime \prime}$ within $30 \%$ uncertainty for various fluids when the contact angle ranges from $0^{\circ}$ to $90^{\circ}$. Although the measurement of the dynamic receding contact angle in practice will require some effort, the implementation of the model after knowing the dynamic contact angle is very straightforward. This model can be used to provide the baseline prediction for a wide range of fluids and operation conditions. Considering the dynamic behaviour of the bubbles, Kandlikar's model for CHF can be understood as the threshold condition where the bubble starts to be stretched sideway when it grows to the specified size (i.e. $\lambda_{C, R T} / 2$ ). There is no direct experimental evidence to show this threshold condition, and 
the choice of the bubble size was not justified. In addition, the experiments reported by Chu et al. (Chu et al., 2014) show that the bubbles start to expand laterally and merge with other bubbles at 73\% CHF.

\section{(iii) Macrolayer dryout models}

The macrolayer dryout model was initially developed by Haramura and Katto (Haramura and Katto, 1983). The proposed physical process is shown in Fig. 2.

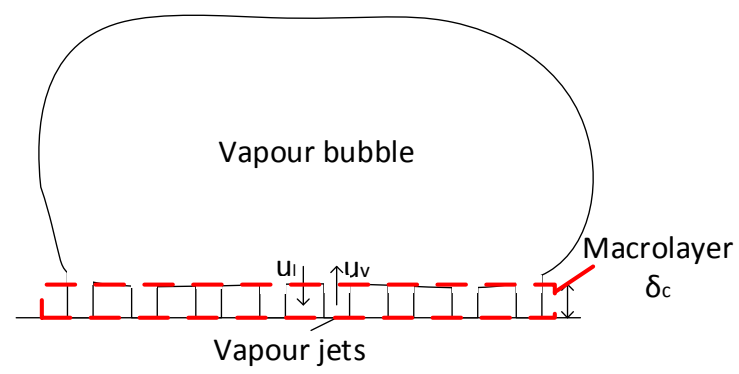

Fig. 2 Vapour structure near heated surface at high heat flux proposed by Haramura and Katto

This group of models assume that large bubbles are hovering above a macrolayer which consists of numerous small vapour jets separated by the thin liquid film. The evaporation of the liquid film in this layer feeds the vapour to the large bubbles above them. The model assumes the CHF occurred when this macrolayer evaporated entirely before the bubble grows large enough to escape from the surface. Therefore, the $q_{C H F}^{\prime \prime}$ can be calculated as the total amount of heat required to evaporate all the liquid films inside the macrolayer. This group of models assumes:

1. The thickness of this macrolayer must be sufficiently small to avoid the $\mathrm{KH}$ instability. As a result, the model assumes the layer thickness $\left(\delta_{c}\right)$ must be proportional to the $\mathrm{KH}$ instability wavelength $\left(\lambda_{K H}\right)$. The original model assumes $\delta_{c}=\lambda_{K H} / 4$. 
2. The bubbles are separated by a distance equals to the most dangerous wavelength $\left(\lambda_{D, R T}\right)$ predicted by the RT instability.

3. The hovering period $\left(\tau_{d}\right)$ needs to be calculated based on the hydrodynamic theory. Haramura and Katto applied the theory suggested by Davidson et al. (Davidson and Schuler, 1960) which was originally developed to calculate the hovering period for a large immersed bubble generated by a small orifice plate.

The $q_{C H F}^{\prime \prime}$ can then be calculated as (Haramura and Katto, 1983):

$q_{C H F, H K}^{\prime \prime}=q_{C H F, Z}^{\prime \prime}\left(\frac{\pi^{4}}{2^{11 \cdot 3^{2}}}\right)^{1 / 16}\left(\frac{A_{v}}{A_{S}}\right)^{5 / 8}\left(1-\frac{A_{v}}{A_{S}}\right)\left\{\frac{\left(\rho_{l} / \rho_{v}\right)-1}{\left((11 / 16)\left(\rho_{l} / \rho_{v}\right)+1\right)^{3 / 5}}\right\}^{5 / 16}$

where $A_{v}$ is the total cross-sectional area of the vapour jets

The Haramura and Katto's model is in good agreement with the Zuber's model when $A_{v} / A_{s}=0.584\left(\rho_{v} / \rho_{l}\right)^{0.2}$.

The Haramura and Katto's model has been revised by many researchers based on the different hovering period and macrolayer thickness models to fit the experimental data better, as detailed in ref. (Fang and Dong, 2016). The main uncertainties of this group of models are coming from the errors in predicting the hovering period and macrolayer thickness, which are also difficult to be measured accurately.

The physical process postulated by this group of models also contradict many experimental observations, especially the experiments designed to measure the dry area underneath the bubbles (Ahn and Kim, 2012; Chu, No and Song, 2013; Chu et al., 2014). For example, visualisations did by Chu et al. (Chu, No and 
Song, 2013)(Chu et al., 2014) show that the macrolayer has been thoroughly dried out at heat flux conditions much lower than the CHF condition.

\section{(iv) Dry spots models}

Dry spots models have been suggested by Yagov in 1988 (Yagov, 1988) in light of the experimental observations that the dry area fraction increases as the heat flux increases in pool boiling. Yagov reckoned that the increase of the dry areas as the heat flux increase could lead to two competing effects. It will enhance the heat transfer due to the high local heat transfer rate from the larger areas of the thin liquid film in the vicinity of the dry spots. On the other hand, it can terminate the nucleate boiling if the wetted area becomes too small to sustain the evaporation rate required at high heat flux. The model assumes the CHF occurs when the liquid evaporation rate is higher than the liquid inflow rate due to the capillary force around the dry spots, as in Fig. 3. By assuming the critical dry spot size is proportional to the bubble departure diameter $\left(D_{d}\right)$, and the interface curvature gradient near the dry spot $(d H / d r)$ is correlated to the macrolayer thickness as: $d H / d r=1 / \delta_{0}^{2}$, Yagov derived the equation used to calculate the heat flux near the dry spots $q_{d s}{ }^{\prime \prime}$ (Yagov, 2014):

$q_{d s}^{\prime \prime}=C_{0} \frac{\sigma h_{l v}}{v} \frac{\delta_{0}}{D_{d}}$

where $C_{0}$ is an empirical constant for fitting the experimental data, $v$ is the kinematic viscosity, $\delta_{0}$ is the liquid film thickness, $D_{d}$ is the bubble departure diameter. 


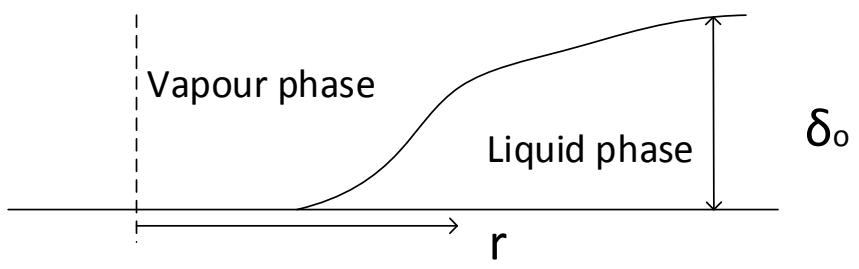

Fig. 3 Schematic of the liquid meniscus at the interface in Yagov's model When the average heat flux is higher than the heat flux near the dry spots (i.e., $\left.q^{\prime \prime}>q_{d s}{ }^{\prime \prime}\right)$, the dry spots will grow and merge together to initiate the $\mathrm{CHF}$ condition. Therefore, the $\mathrm{CHF}\left(q_{C H F, Y}^{\prime \prime}\right)$ is equal to $q_{d s}{ }^{\prime \prime}$. Separate semi-empirical models for predicting $D_{d}$ and $\delta_{0}$ are used to calculate $q_{C H F, Y}^{\prime \prime}$ in different pressure regimes (Yagov, 2014).

Yagov postulates that in low reduced pressure limit (i.e. $P \ll P_{c}$, where $P_{c}$ is the critical pressure), the dominant heat removal mechanism is the convective heat transfer caused by the vapour generation; while in high reduced pressure limit, the dominant mechanism is the liquid evaporation at the boundaries of the dry spots. Using various semi-empirical correlations, he suggested the following Equations to calculate the heat flux in CHF (Yagov, 2014):

$$
\begin{aligned}
& q_{C H F, Y, L P}^{\prime \prime}=0.5 \frac{h_{l v}^{81 / 55} \sigma^{9 / 11} \rho_{v}^{13 / 110} k^{7 / 110} g^{21 / 55} f(\mathrm{Pr})}{v^{1 / 2} c_{p}^{3 / 10} R_{i}^{79 / 110} T_{s}^{21 / 22}} ; f(\mathrm{Pr})=\left(\frac{\mathrm{Pr}^{9 / 8}}{1+2 \operatorname{Pr}^{1 / 4}+0.6 \mathrm{Pr}^{10 / 24}}\right)^{4 / 11} \\
& q_{C H F, Y, H P}^{\prime \prime}=0.06 h_{l v} \rho_{v}^{0.6} \sigma^{0.4}(g \Delta \rho / \mu)^{0.2} \\
& q_{C H F, Y}^{\prime \prime}=\left(q_{C H F, Y, L P}^{\prime \prime}{ }^{3}+q_{C H F, Y, H P}^{\prime \prime}\right)^{1 / 3}
\end{aligned}
$$

where $k$ is the thermal conductivity, $c_{p}$ is the heat capacity, $R_{i}$ is the gas constant, $T_{s}$ is the surface temperature, Pr is the Prandtl number.

Eq. (8) is constructed so that the term with smaller value loses its contribution quickly in comparison with the larger one. Yagov believes the $q_{C H F, Y}^{\prime \prime} \approx q_{C H F, Y, H P}^{\prime \prime}$ 
in most practical cases where the pressure is in medium to high range. Direct visualization results reported by various researchers (Theofanous et al., 2002; Ahn and Kim, 2012; Chu, No and Song, 2013; Chu et al., 2014; Kim, Song and Kim, 2016) confirm the existence of the large dry spot near the CHF conditions, as in Fig. 4.
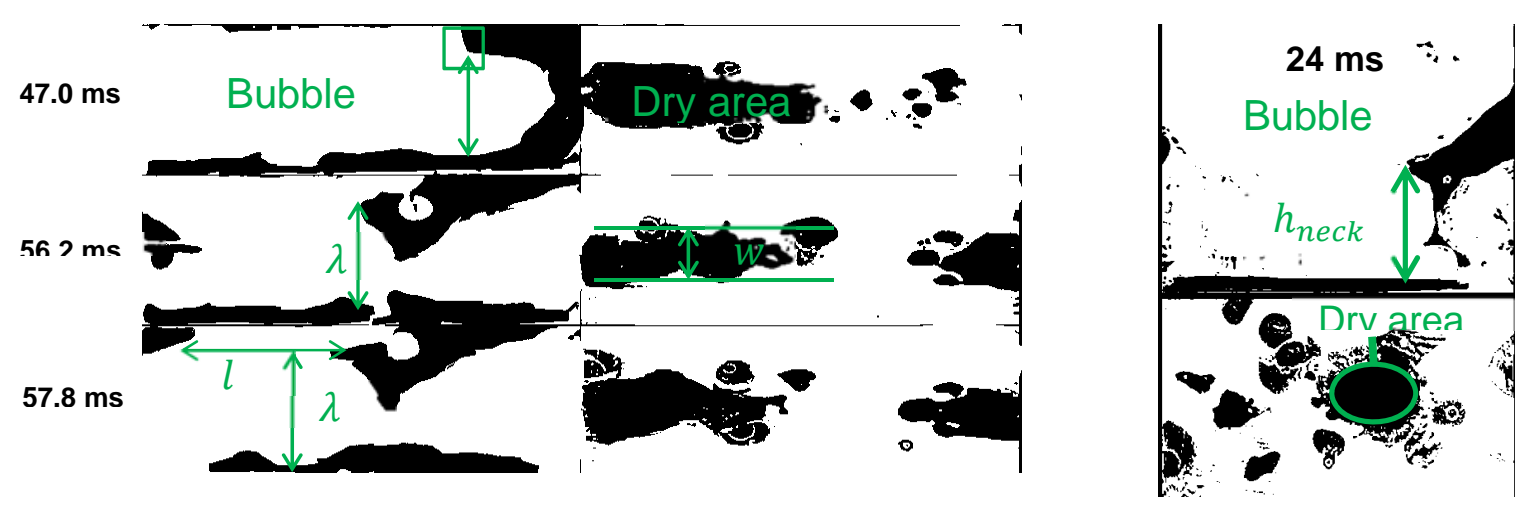

(a) Chu et. al. (Chu, No and Song, 2013)

(b) Chu et. al. (Chu et al., 2014)

Fig. 4 Bubble interface in CHF conditions. (a) Left-hand images show the side view of bubbles (white area), and the right-hand images show the distribution of the dry areas (black area) in heating surface. (b) The top image shows the side view of the bubble (white area), and the bottom images show the distribution of the dry areas (black area) in heating surface. Images are obtained by post-processing (contrast enhancing and binary image conversion) the photos reported in literature

However, the static threshold condition to initiate the CHF described by Yagov contradicts the periodic bubbling process observed in experiments (Chu, No and Song, 2013), as represented in Fig. 4. As it can be seen from Fig. 4, the dry patches do exist underneath the coalesced bubbles during the bubble growth and merging process. However, the area of these dry patches shrinks periodically during the bubble departure process (i.e. stage (c) - (g) in Fig. 5) in 
CHF conditions instead of increasing monotonically as suggested by Yagov's model.

There are two different types of bubble behaviour when dry spots have been observed experimentally during the high heat flux nucleate boiling regime (Chu, No and Song, 2013; Chu et al., 2014)(Kim, Song and Kim, 2016), as shown in Fig. 5.

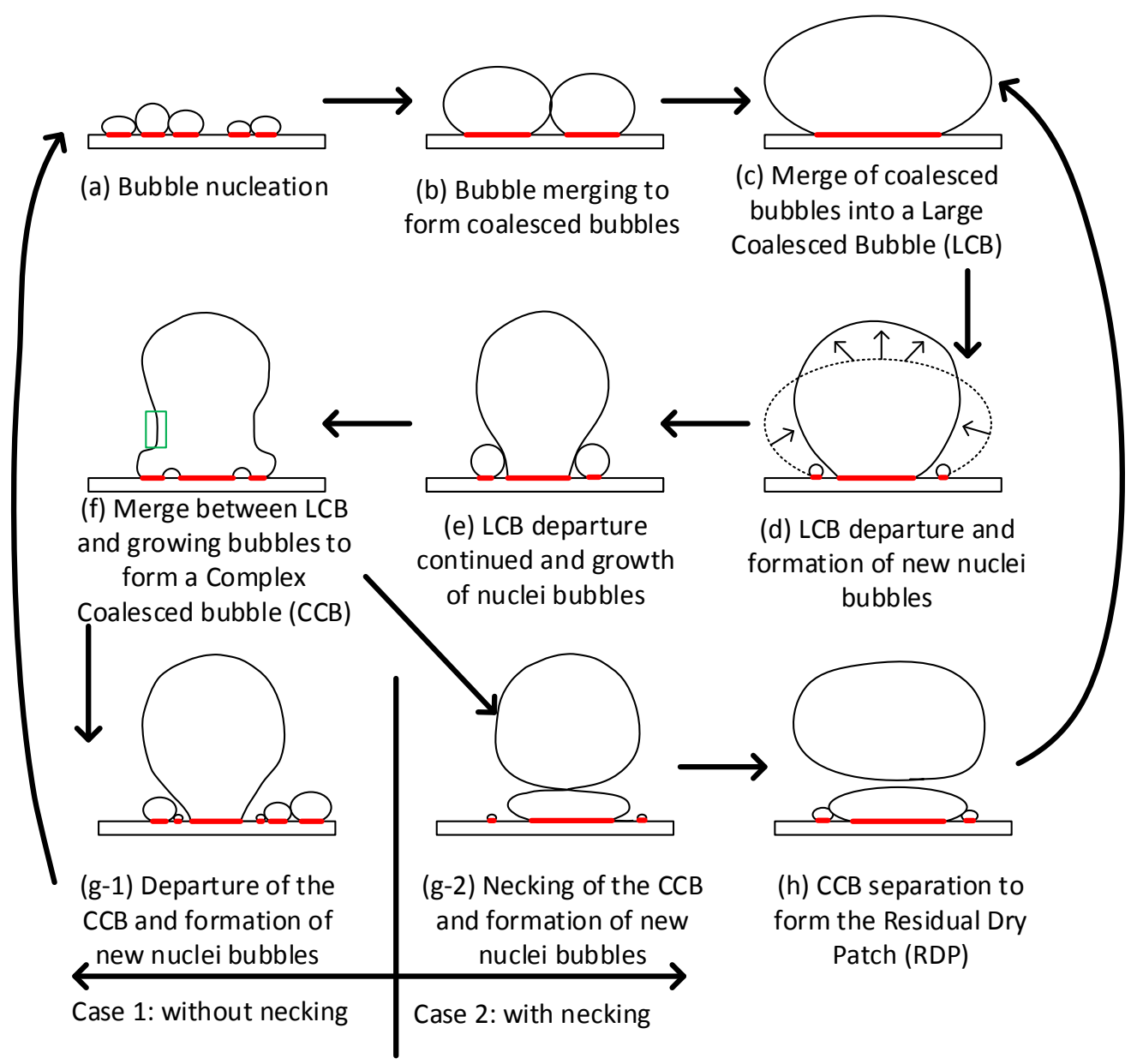

Fig. 5 The two-dimensional periodic behaviour of bubbles in high heat flux nucleate boiling conditions. The bold dashed line indicates the dry area. The small box in stage (f) highlights the liquid-vapour interface where the large bubble merges with the growing nuclei bubbles 
The first type is represented in case 1 in Fig. 5 when the dry area below the bubble will appear and disappear periodically through the bubble growth and detachment process. Case 2 in Fig. 5 represents the second type when the bubble necking and separation process appears (stage (g-2) and stage (h)), which then left a residual bubble on the surface. In case 2, the dry area initially shrinks during the bubble detachment process and then grows as the residual bubble leftover from the separation process starts to grow. As shown in the visualisation results reported by Chu et al. (Chu et al., 2014), when the heat flux is significantly lower than the CHF condition, case 1 is dominant; as heat flux increases, case 2 starts to appear more frequently. It is important to note that conditions local to any individual bubble may lead to the departure of bubbles with or without necking (g-1 or g-2) depending on the local interactions. As heat flux increases, the statistical likelihood of bubble departure through necking (g2) increases. Below the critical heat flux, the case 1 bubble behaviour can still happen from time to time to rewet the surface. At or above the $\mathrm{CHF}$, the case 2 bubble behaviours repeated consistently so that although the hot spots underneath the bubbles shrink and expand periodically, they never disappear. These hot spots are named Irreversible Hot Spot (IHS). The surface temperature on the IHS then increases with time, which will heat up the adjacent liquid to superheat condition to trigger more evaporation. As a result, the average size of the IHS increases over time until it permanently covers a substantial portion or even the whole heating surface to initiate the CHF condition. This description of the net rate of change of dry spot area does not preclude occasional rewetting of the surface in both the spatial and time 
domains which can be expected as local conditions are statistically variable. Experiments did by Chu et al. (Chu et al., 2014) show that the IHS does not exist in 95\% CHF heating condition but appears in the early stage of the CHF heating condition.

In summary, the observed physical process and quantitative measurements reported in the literature (Theofanous et al., 2002; Ahn and Kim, 2012; Chu, No and Song, 2013; Chu et al., 2014) show that the formation of the IHS can initiate the CHF condition. This mechanism is compatible with the CHF initiate mechanisms for nano-structured surfaces reported by Dhillon et al. (Dhillon, Buongiorno and Varanasi, 2015). Dhillon et al. reported that the CHF on nanotextured surfaces is higher than that on the flat surface because the capillary forces on the nano-textured surfaces will induce the liquid imbibition to rewet the surface. Through both experiments and modelling, Dhillon et al. found that the $\mathrm{CHF}$ is initiated when the liquid evaporation rate is higher than the liquid imbibition rate. In other words, the CHF is initiated when the hot dry spot cannot be rewetted causing the IHS to appear.

The idea that the CHF can be triggered by IHS is not new and has been explored by many researchers to model the CHF condition. The work done by Unal et al. (Unal, Daw and Nelson, 1992) was the first attempt to find the conditions where the IHS can exist. They built up a transient heat conduction model to first calculate the required dry patch size so that the temperature at the centre of the dry patch can reach the homogeneous nucleation temperature $\left(345^{\circ} \mathrm{C}\right.$, water) to avoid any further rewetting process. Their calculation 
indicates the required dry patch size is much bigger than the measured value which leads to the conclusion that the temperature at the centre in $\mathrm{CHF}$ conditions must be lower than the homogeneous boiling temperature. They then applied the same model to calculate the temperature at the centre of the dry patch and the required dry patch size when the modelled heat flux value matches a measured CHF value. Their calculations, based on different initial macrolayer thickness before drying out, suggest the temperature varies from $157^{\circ} \mathrm{C}$ to $180^{\circ} \mathrm{C}$ when the initial macrolayer thickness changes from 0 to $11 \mu \mathrm{m}$, which agree with the measured transition-film boiling temperature on the surface where the contact angle is $60-70^{\circ}$. Such an agreement suggests their heat transfer models can predict the surface temperature reasonably well but does not explain the cause of the appearance of IHS. Choi et al. (Choi, No and Kim, 2016) also proposed another CHF model which is based on the postulation that the IHS is produced when the dry patch peripheral temperature reaches the Leidenfrost temperature so that they cannot be rewetted even when the bubble is detached from the heating surface. However, Theofanus et al. (Theofanous et al., 2002) and Kim et al. (Kim, Song and Kim, 2016) measured the surface temperature of the irreversible dry spot when it was just created in $\mathrm{CHF}$ conditions and found the surface temperature is between $120-160{ }^{\circ} \mathrm{C}$, which is much lower than the Leidenfrost temperature of the coolant $\left(\sim 200^{\circ} \mathrm{C}\right)$. Therefore, all the existing models do not capture the physical process accurately.

In the visualisation results reported by Chu et al. (Chu, No and Song, 2013), as in Fig. 4 and Fig. 5, the IHS is a result of the disintegration of the Complex 
Coalesced Bubble (CCB) - stage (g-2) to stage (h)). The disintegration is coming from the necking process, as shown in stage (f) to stage (g-2) in Fig. 5. Similar necking process has also been reported by Ahn and Kim through direct visualisation (Ahn and Kim, 2012). Based on the observation that the best fit models to predict the heat flux in CHF condition are semi-empirical hydrodynamic instability models despite their bold and controversial assumption, we postulate that the necking process could be caused by the hydrodynamic instability of the CCB.

Through more rigorous consideration of the hydrodynamic instability of the CCB, a new model used to predict the heat flux in CHF conditions has been developed and will be presented in next section.

\section{The new hydrodynamic instability irreversible hot spot model}

As detailed in the previous section, the CHF condition can be triggered by the appearance and expansion of IHS due to the consistent bubble separation process (case 2 in Fig. 5). The new model for predicting CHF is developed based on the following physical process, which represents the spatial and timeaveraged cyclic bubble behaviours in CHF condition:

1. The hydrodynamic instability of the liquid-vapour interface initiates the large coalesced bubble separation process, as shown in Fig. 4 and Fig. 5 from the stage (f) to stage $(g-2)$. It should be noted that such a separation process does not depend on whether the top part of the coalesced bubble is isolated or merged with the adjacent bubbles, as 
long as there is a clearly defined interface where the instability induced separation process occurs.

2. The separation processes occur consistently when the heat flux condition can consistently trigger the hydrodynamic instability, which produces the Irreversible Hot Spot (IHS). The IHS will grow with time and eventually cover a substantial portion of the heating surface to trigger the $\mathrm{CHF}$.

To predict when and where the hydrodynamic instability occurs requires detailed analysis of the liquid-vapour interface which is close to the heating surface. According to the experimental visualization of the bubble interface in boiling (Chu, No and Song, 2013), the most vulnerable vapour-liquid interface just before the necking process could be the interface highlighted in the rectangular box in Fig. 4 where the large bubble is in the process of merging with the growing new-born bubbles while detaching from the surface. The convection and turbulence induced by the bubble merging and departure can initiate the instability by disturbing the interface. In addition, the cross-sectional area in this region is the smallest, producing the highest relative velocity between the liquid flow and the vapour flow, making it a likely point for initiating the instability.

This interface can be approximated as a vertical interface with horizontal flow perturbation (i.e. the vapour is mainly flowing upward, and the bubble separation is parallel to the heating surface, as shown in Fig. 6.). The instability criterion for a vertical interface subject to horizontal perturbations due to both surface tension force and dynamic pressure force in inviscid flow is a special case of the $\mathrm{KH}$ instability. 


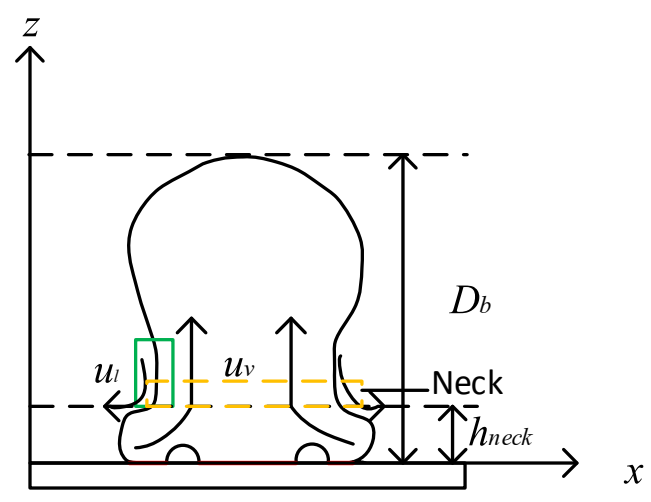

Fig. 6 Hydrodynamic stability during the bubble merging process, i.e. stage (f) in Fig.5

The instability criterion is indicated by Eq. (1). The most vulnerable condition, where the initial perturbation grows at the fastest rate, can be calculated by Eq.

(9) through instability analysis as:

$u_{D}=\left|\bar{u}_{l}-\bar{u}_{v}\right| \approx \bar{u}_{v}=\left[\frac{6 \pi \sigma\left(\rho_{l}+\rho_{v}\right)}{\rho_{l} \rho_{v} \lambda_{D, K H}}\right]^{1 / 2}=\sqrt{3 / 2} u_{C}$

Therefore, the instability occurs when

$u_{c} \leq \bar{u}_{v} \leq \sqrt{3 / 2} u_{c}$

When sensible heat is negligible in high heat flux condition, $\bar{u}_{v}$ at height $z$ can be calculated as:

$\bar{u}_{v}(z)=\frac{\bar{q}_{w}^{\prime \prime}}{\rho_{v} h_{l v}} \cdot\left(\frac{A_{\text {base }}}{A(z)}\right)$

where $\bar{q}_{w}^{\prime \prime}$ is the time-averaged heat flux input, $A_{\text {base }}$ is the total base area contribute to the evaporation during the bubbling event and $A(z)$ is the crosssectional area of the bubble as a function of height $z$. 
Eq. (10) and (11) can be used to decide whether the instability occurs or not. When $q_{w}^{\prime \prime}$ is relatively small, $\bar{u}_{v}$ will be relatively small which requires $\lambda_{K H}$ to be relatively large. If $\lambda_{K H}$ is bigger than the coalesced bubble diameter $\left(D_{b}\right)$, bubble separation would not happen, and the bubble will eventually depart from the surface. Consequentially, the surface will be fully rewetted and the IHS would not appear. As $q_{w}^{\prime \prime}$ is further increased, the required $\lambda_{K H}$ is reduced, and the bubble separation at a point away from the wall starts to occur, i.e. when $\lambda_{K H}<$ $D_{b}$. Eq. (10) and (11) can also be used to decide where the instability will occur. Considering the flow condition depicted in Fig. 6 (the same as the stage (f) in Fig. 5), the instability is unlikely to occur below the neck because $\bar{u}_{v}$ is relatively small, due to the large $A(z)$, while the required $u_{c}$ is relatively large, due to the small perturbation wavelength $\left(\lambda_{K H} \leq h_{n e c k}\right)$. At the neck, $\bar{u}_{v}$ achieves the maximum value and the flow perturbation is also the strongest due to the merging and detaching process. Therefore, this is the region where the instability is most likely to occur if the heat flux is big enough to satisfy the condition defined in Eq. (10). When the heat flux is not big enough to satisfy Eq. (10), the surface tension will act to round the merged bubble so that the neck region moves downward to the bottom of the bubble, as shown in the stage (g1) in Fig. 5. The instability is unlikely to occur anywhere near the bottom region due to the reduced $\bar{u}_{v}$ and increased $u_{c}$. The bubble detaching process is then continued and the IHS will not appear.

By combining Eq. (10) and (11), the new model used to predict the timeaveraged heat flux in CHF can be written as 
$\frac{\bar{q}_{C H F, I H S}^{\prime \prime}}{\bar{q}_{w, c}^{\prime \prime}}=C \in[1, \sqrt{3 / 2}], q_{w, c}^{\prime \prime}=\sqrt{\frac{2 \pi \sigma\left(\rho_{l}+\rho_{v}\right)}{\rho_{l} \rho_{v} h_{\text {neck }}}} \rho_{v} h_{l v} \frac{A_{\text {neck }}}{A_{\text {base }}}$

where $C$ is a constant. $C=1$ is corresponding to the critical $\mathrm{KH}$ instability condition and $C=\sqrt{3 / 2}$ is corresponding to the most dangerous $\mathrm{KH}$ instability condition. Therefore, its value is depending on the perturbation strength (e.g. flow turbulence) and the interface resistance to perturbation (e.g. surface tension of the fluids, or the heating surface orientation).

The experimental results reported by Chu et al. (Chu, No and Song, 2013) and Chu et al. (Chu et al., 2014), as shown in Fig. 4, provide useful data to test Eq. (12). Taking into account the setup of the side-view camera, the data extracted from Fig. 4 (a) are:

$\lambda=8 \mathrm{~mm} ; l=4.6 \mathrm{~mm} ; w=1.9 \mathrm{~mm} ; h_{n e c k}=\lambda / \cos \left(39^{\circ}\right) \approx 11.1 \mathrm{~mm} ;$

$A_{\text {neck }} \approx l \times w=4.6 \times 1.9=8.74 \mathrm{~mm}^{2} ; A_{\text {base }}=W \times L=2.7 \times 27.1=73.17 \mathrm{~mm}^{2}$ where $39^{\circ}$ is the camera inclination angle; $W$ is the heater width; $L$ is the heater length. Considering the maximum bubble length will be limited by the RT instability and the actual heater is much longer than $\lambda_{D, R T}, L$ is taken as $\lambda_{D, R T}$. Similarly, the data extracted from Fig. 4 (b) are: $h_{\text {neck }} \approx 6.2 \mathrm{~mm} ; A_{\text {neck }} \approx 12.38 \mathrm{~mm}^{2} ; A_{\text {base }}=W \times L=8 \times 27.1=217.6 \mathrm{~mm}^{2}$ The comparison between the measured parameters and the CHF predicted by Eq. (12) based on the measured parameters are summarised in Table 1 . The comparison suggests the constant $C$ in Eq. (12) is close to 1. Therefore, $C$ will be taken as 1 to calculate the CHF conditions on this paper.

To calculate the heat flux in CHF conditions, models for predicting $h_{\text {neck }}$ and $A_{\text {neck }} / A_{\text {base }}$ in different boiling conditions are required. These models can be 
constructed by considering the detailed bubble nucleation and merging process, as depicted in Fig. 5 and Fig. 7. A similar bubble merging process has been reported by Chu et al. (Chu, No and Song, 2013). Please note that Fig. 5 and Fig. 7 represents the cross-sectional view of the spatial and time averaged cyclic bubble behaviour.

Table 1 Comparison between experimental data and Eq. (12)

\begin{tabular}{|c|c|c|c|c|c|c|}
\hline Paper & $\begin{array}{c}h_{\text {neck }} \\
(\mathrm{mm})\end{array}$ & $\begin{array}{c}A_{\text {neck }} \\
\left(\mathrm{mm}^{2}\right)\end{array}$ & $\begin{array}{c}A_{\text {base }} \\
\left(\mathrm{mm}^{2}\right)\end{array}$ & $\begin{array}{c}q_{w, C H F}^{\prime \prime} \\
\left(\mathrm{kW} / \mathrm{m}^{2}\right)\end{array}$ & $\begin{array}{c}q_{w, C}^{\prime \prime} \\
\left(\mathrm{kW} / \mathrm{m}^{2}\right)\end{array}$ & $\frac{q_{w, C H F}^{\prime \prime}}{q_{w, C}^{\prime \prime}}$ \\
\hline $\begin{array}{c}\text { Chu et al. (Chu, No } \\
\text { and Song, 2013) }\end{array}$ & 11.1 & 8.74 & 73.17 & 1351 & 1206 & 1.12 \\
\hline $\begin{array}{c}\text { Chu et al. (Chu et al., } \\
\text { 2014) }\end{array}$ & 6.2 & 12.38 & 216.8 & 826 & 769 & 1.07 \\
\hline
\end{tabular}

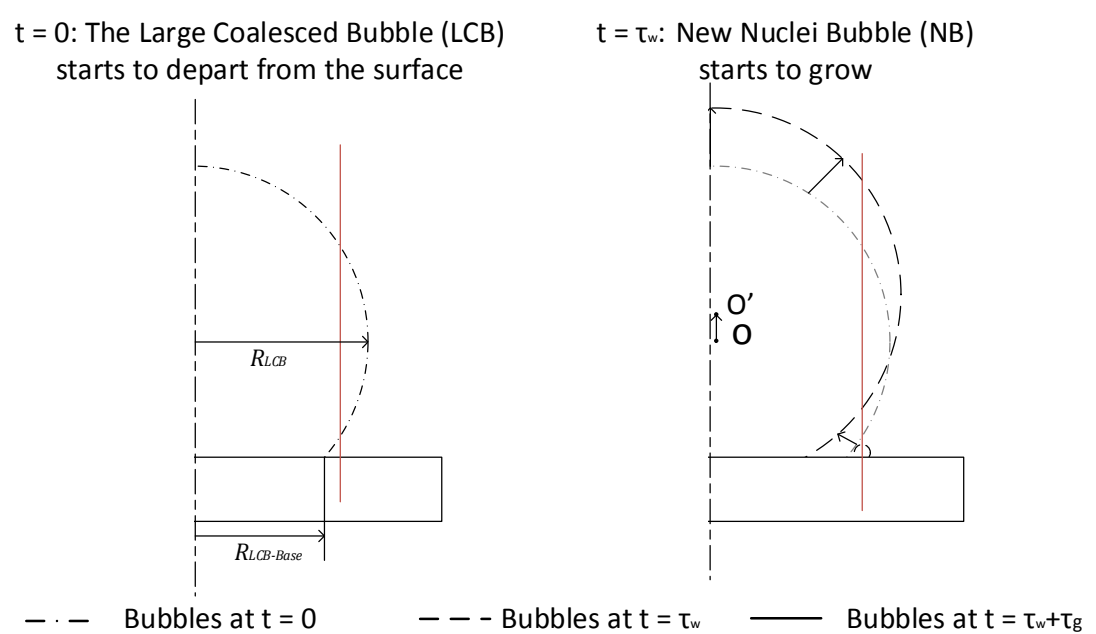

$t=\tau_{w}+\tau_{g}: N B$ starts to merge with LCB

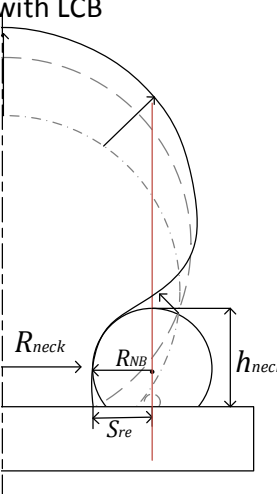

Fig. 7 Cross-sectional view of the threshold condition for the bubble merging (spatial and temporal averaged behaviour)

As the Large Coalesced Bubble (LCB) is detaching from the surface (stage (d) in Fig. $5 ; t=0$ in Fig. 7), the bubble contact line is receding and part of the dry surface is rewetted. After a certain period of waiting time $\left(\tau_{w}\right)$, new Nuclei 
Bubbles (NBs) start to appear on the surface originally covered by the microlayer. These NBs continue to grow while the LCB is detaching. When the heat flux is relatively low, the NBs cannot grow fast enough to be able to merge with the detaching LCB to feed the extra vapour to induce the instability (i.e. $\bar{u}_{v}<\bar{u}_{c}$ ) so the LCB will be fully detached from the surface, i.e. case 1 in Fig. 5. As the heat flux increases, the NBs will grow faster and they start to be in touch with the LCB (stage (e) in Fig. 5) to initiate the bubble merging process (stage (f) in Fig. 5, $t=\tau_{w}+\tau_{g}$ in Fig. 7) to form the Complex Coalesced Bubble (CCB). During the merging process, the height of NBs seems changed very little, as shown in Fig. 4 (a), possibility because the evaporated vapours are redistributed within the CCB and NBs are much smaller than LCB. As shown in Fig. 7, the line of merging marks the neck of the CCB. When the combination of the imposed heat flux and the neck area fits the instability criteria (i.e. Eq. (12)), necking consistently occurs and it will eventually trigger the CHF, i.e. case 2 in Fig. 5. Therefore, the necking process is triggered by the bubble merging process. In real system, bubbles can be of different sizes and be distributed non-uniformly so there is no fixed CHF condition. The model presented in this paper is based on the spatial and time averaged cyclic bubble behaviour, as in Fig. 5 and Fig. 7, so will predict the average CHF condition over time.

According to the physical process described above, $h_{\text {neck }}$ in Eq. (12) can be calculated according to existing bubble growth models if the growth time $\left(\tau_{g}\right)$ is known. $\tau_{g}$ can be determined by the condition to initiate the merging process to produce the $\mathrm{CCB}$, as detailed in Fig. 7. The bubble merging process is shown to occur when one bubble grow big enough to reach the base of the other bubble 
(three phrase contact line)(Chu, No and Song, 2013), i.e. $R_{N B}\left(\tau_{g}\right)=S_{r e}\left(\tau_{w}+\right.$ $\tau_{g}$ ) where $S_{r e}$ is the distance between the nucleation sites and the bubble base. According to Fig. 7, the height and radius of the bubble neck can be calculated by Eq. (13).

$$
\begin{aligned}
& h_{\text {neck }}\left(\tau_{g}\right)=R_{N B} \cdot\left(1+\cos \beta_{r e}\right) ; \\
& R_{\text {neck }}\left(\tau_{g}\right)=R_{L C B, \text { base }}\left(\tau_{g}\right)
\end{aligned}
$$

where $\beta_{r e}$ is the receding contact angle

The $R_{N B}\left(\tau_{g}\right)$ can be calculated using existing bubble growth models. The NB bubble can be treated as isolated bubble before merging with the LCB so many different bubble growth models have been suggested and reviewed in existing literatures (van Stralen et al., 1975; Zijl, 1978; Carey, 2008). If the very short period of inertial-control bubble growth regime is ignored, the bubble growth models in the heat transfer controlled regime in the saturated condition can be well represented by Eq. (14) no matter whether the heat is from the relaxation layer above the bubble dome (Zuber, 1961; Mikic, Rohsenow and Griffith, 1970) or from the microlayer underneath the bubble (Cooper, 1969; van Stralen et al., 1975).

$R_{N B}\left(\tau_{g}\right)=\gamma \cdot\left(\alpha_{l} \tau_{g}\right)^{n} ; \gamma=C_{1} \cdot J a ; J a=\frac{\Delta T_{s} \cdot C_{p, l} \cdot \rho_{l}}{h_{l v} \cdot \rho_{v}} ; \Delta T_{s}=T_{s}-T_{s a t}$

where $\gamma$ is the bubble growth rate coefficient; $C_{1}$ is a coefficient depending on the pressure; $J a$ is the Jacob number; $\alpha$ is the thermal diffusivity; $\Delta T_{S}$ is the surface superheat. 
The exponent, $n$, was reported in most models and experiments to be 0.5 but has been reported to vary between 0.3 and 0.7 [20] - (Cooper and Lloyd, 1969). The reported growth coefficient $C_{1}$ in literature has a wide range. The maximum value of $C_{1}$ is corresponding to the growth of a free spherical bubble in an initially uniform superheated infinite liquid and is shown to be equal to 1.954 (Plesset and Zwick, 1954). $C_{1}$ in the microlayer evaporation models developed by Cooper and Lloyd (Cooper and Lloyd, 1969), Ouwerkerk (van Ouwerkerk, 1971) and Stralen et al. (van Stralen et al., 1975) is a function of the liquid Prandtl number and is equal to $1.71,1.41$ and 0.40 respectively in atmospheric pressure. The predicted bubble diameter from the Cooper and Lloyd's model agree well with the experimental data on a flat surface when the bubble is small $(<0.5 \mathrm{~mm})$ but it overestimates the bubble diameters significantly when the bubble becomes larger (Cooper, 1969). The bubble diameters predicted by Ouwerkerk's model are larger than the observed Heptane and Benzene diameter on a flat surface by less than $10 \%$. Therefore, the actual value of $C_{1}$ in atmospheric pressure is likely to be somewhere between 1.4 and 1.7. There is no comparison between the predicted bubble diameter from the model developed by Stralen et al. (van Stralen et al., 1975) and the experiments. The $C_{1}$ in models proposed by Stralen et al. is assuming pure laminar boundary layer so it likely to be the lower limit of $C_{1}$. The effect of $C_{1}$ on the proposed model will be presented and discussed in more detail in next section.

$T_{S}$ can be calculated using the established nucleation boiling correlation, such as the one suggested by Rohsenow (Rohsenow, 1951), i.e. Eq. (15). Eq. (15) 
suggests that $\Delta T_{S}$ is proportional to $q_{w}^{\prime \prime 1 / 3}$ so it will not be sensitive to the small change of $q_{w}{ }^{\prime \prime}$.

$\Delta T_{S}=\left[\left(\frac{q_{C H F, I H S}^{\prime \prime}}{\mu h_{l v}}\right) \cdot\left(g \cdot \frac{\rho_{l}-\rho_{v}}{\sigma}\right)^{-0.5}\right]^{\frac{1}{3}} \cdot \frac{C_{s f} \cdot h_{l v} \cdot P r_{l}^{m}}{C_{P, l}}$

where the value of the coefficients $C_{s f}$ and $m$ depend on the combination of fluids and surface and are well-documented on literature (Pioro, 1998; Carey, 2008).

The $R_{\text {base }}\left(\tau_{g}\right)$ can be predicted by considering the forces acting on the LCB. Assuming the dominant forces in sub-atmospheric and near atmospheric pressure conditions are the inertial force due to bubble growth and buoyancy, the force balance Equation can be written as Eq. (16) as proposed by Zijl (Zijl, 1978).

$\frac{d}{d t}\left(\frac{2}{3} \pi \cdot \rho_{l} \cdot R_{L C B}^{3}(t) \cdot \dot{Z}(t)\right)=\frac{4}{3} \pi\left(\rho_{l}-\rho_{v}\right) \cdot R_{L C B}^{3}(t) \cdot g$

where $R_{L C B}(t)$ is the radius of the LCB, $\dot{z}$ is the velocity of the centroid of the bubble. Eq. (16) was solved by Zijl when $R_{L C B}(t)=\gamma_{L C B} \cdot\left(\alpha_{l} t\right)^{0.5}($ Zijl, 1978) as in Eq. (17).

$$
\begin{aligned}
& R_{L C B, b a s e}(t)=\left(\gamma_{L C B}^{2} \alpha_{l} t-\frac{4 g^{2}}{25} t^{4}\right)^{0.5} ; R_{d}=\left(\frac{5 \gamma_{L C B}^{4} \alpha_{l}^{2}}{2 g}\right)^{1 / 3} ; \\
& t_{d}=\left(\frac{5 \gamma_{L C B}}{2 g}\right)^{2 / 3} \alpha_{l}^{1 / 3} ; t_{o}=\frac{t_{d}}{2^{2 / 3}}
\end{aligned}
$$


where $R_{d}$ is the bubble departure diameter; $t_{d}$ is the departure time; and $t_{o}$ is the time when the bubble starts to detach (i.e. $R_{L C B, b a s e}$ has reached its maximum value).

Experiments by Sakashita and Ono (Sakashita and Ono, 2009) suggests that the LCB departure diameters in CHF under different pressure conditions are close to the critical wavelength of RT instability (i.e. $\lambda_{c, R T}$ ). This result combined with Eq. (17) suggested that $\gamma_{L C B}$ can be calculated by Eq. (18).

$\gamma_{L C B}=\left(\frac{(\lambda c, R T / 2)^{3} \cdot 2 g}{5 \cdot \alpha_{l}^{2}}\right)^{1 / 4}$

Following the same steps suggested by Zijl, Eq. (16) can be solved for more general conditions when $R_{L C B}(t)=\gamma_{L C B} \cdot\left(\alpha_{l} t\right)^{n}$. The solutions can be summarized as in Eq. (19).

$$
\begin{aligned}
& R_{L C B, b a s e}(t)=\left(\gamma_{L C B}^{2} \cdot\left(\alpha_{l} t\right)^{2 n}-\left(\frac{g}{3 n+1}\right)^{2} t^{4}\right)^{0.5} ; \gamma_{L C B}=\left(\frac{(\lambda c, R T / 2)^{(2-n) / n} \cdot g}{(3 n+1) \cdot \alpha_{l}^{2}}\right)^{\frac{n}{2}} ; \\
& t_{d}=\left(\frac{\gamma_{L C B \cdot \alpha_{l}^{n}(3 n+1)}}{g}\right)^{1 /(2-n)} ; t_{0}=t_{d} \cdot\left(\frac{n}{2}\right)^{1 /(4-2 n)}
\end{aligned}
$$

According to the process described in Fig. 7, $t_{o}$ in Eq. (17) and (19) is corresponding to $t=0$ in Fig. 7. Similar to other heterogonous bubble inception criteria, the NB is likely to occur inside a superheated microlayer where there is no significant evaporation on the interface between the microlayer and the LCB so that the majority of the heat within the superheated microlayer goes into the bubble nucleus. The typical superheated layer thickness in different heat flux conditions has been measured by Wiebe and Judd (Wiebe and Judd, 1971) and 
their results show that the superheated layer thickness in pool boiling is consistently to be about $1 \mathrm{~mm}$. Considering their measurements cover a wide range of heat flux condition and the heat can be transported more effectively through the interface between the superheated liquid layer and LCB, in current model, the NB is assumed to occur in position when the microlayer thickness is $1 \mathrm{~mm}$. The geometrical analysis for a spherical LCB, as in Fig. 7 suggests:

$$
S_{r e}\left(\tau_{w}+\tau_{g}\right)=\sqrt{\left(\frac{R_{L C B, b a s e}}{\sin \beta_{\text {stat }}}\right)^{2}-\left(\frac{R_{L C B, \text { base }}}{\tan \beta_{\text {stat }}}-0.01\right)^{2}}-R_{L C B, \text { base }}\left(t_{0}+\tau_{w}+\tau_{g}\right)
$$

where $\beta_{\text {stat }}$ is the static contact angle

Therefore, the last final piece of information is the waiting time $\tau_{w}$. Based on the bubble inception condition described by Davis and Anderson (Davis and Anderson, 1966) and the transient heat conduction Equation to semi-infinite region, $\tau_{w}$ can be calculated with Eq. (21). The detailed derivation procescs is shown in Appendix 2. In saturated boiling, $\tau_{w}$ is negligibly small compared to the bubble growth time $\tau_{g}$ so can be ignored.

$$
\begin{aligned}
& \tau_{w}=\left[\left(T_{w, c}-T_{f}\right) \cdot \frac{k_{l}}{2 \cdot q_{w}^{\prime \prime}}\right]^{2} \cdot\left(\frac{\pi}{\alpha_{l}}\right) ; \\
& T_{w, c}=T_{s a t}+\frac{R T_{s a t}^{2}}{h_{l v}} \ln (1+\varepsilon) /\left[1-\frac{R T_{s a t}}{h_{l v}} \ln (1+\varepsilon)\right]+q_{w}^{\prime \prime} \cdot \frac{y^{\prime}}{k_{l}} ; \\
& \varepsilon=\frac{2 \sigma \cdot(1+\cos (\beta))}{P \cdot y^{\prime}} ; y^{\prime}=\frac{1+\cos (\beta)}{P}+\left[\left(\frac{(1+\cos (\beta)) \sigma}{P}\right)^{2}+\frac{2(1+\cos (\beta)) k_{l} \sigma T_{s a t}}{q_{C H F, I H S}^{\prime \prime} h_{l v} \rho_{v}}\right]^{0.5}
\end{aligned}
$$

In summary, different parameters in CHF condition, including the heat flux, the wall superheat, the instability wavelength and the bubble growth rate can be 
decided by solving Eq. $(13-21)$ simultaneously. The required inputs for the model are:

1. The base area $A_{\text {base }}$ in Eq. (12). When the heater size is larger than the most dangerous wavelength predicted by the RT instability (i.e. $\lambda_{D, R T}$ in Eq. (3)), the bubble base area will be ultimately limited by the RT instability so $A_{\text {base }}=\pi \cdot \lambda_{D, R T}^{2} / 4$. When the heater size is smaller than $\lambda_{D, R T}$, the actual heater area will be used. The effect of heater size and heater geometry will be further discussed in next section.

2. The bubble growth coefficient $C_{1}$ in Eq. (14). This is used to count for the difference in the bubble growth rate when the system pressure is changed. Its exact value is depending on the property of the superheated liquid layer around the bubbles, such as its excessive temperature and the size. It likely to be between 1.4 and 1.7 for the horizontal surface at atmospheric pressure, as suggested in literature (Cooper and Lloyd, 1969)(van Ouwerkerk, 1971).

3. The static contact angle $\beta_{\text {stat }}$ in Eq. (20) and the receding contact angle $\beta_{r e}$ in Eq. (13). This is used to count for the difference in bubble geometry when the surface wettability is changed. In calculations done for this paper, $\beta_{r e}$ is assumed to be equal to $\beta_{\text {stat }}$. The effect of changing contact angle will be further discussed in next section.

4. $C_{s f}$ in Eq. (15) which is used to count for the difference in surface temperature when using different liquid-surface combination. Its value is well documented in literature (Pioro, 1998; Carey, 2008). 
There are multiple solutions to this equation sets since the heat flux larger than CHF can still satisfy the criteria described by Eq. (12). Therefore, the CHF condition is corresponding to the solution with the lowest heat flux value.

\section{Results and discussions}

The effect of different inputs, as layout in the previous section (i.e. $A_{\text {base }}, C_{1}$, $\beta$, and $C_{s f}$, has been investigated by applying the proposed IHS model to different boiling conditions. The results, together with their comparison to the experimental data reported in literature, are presented and discussed here. Throughout the calculation, the constant $C$ in Eq. (12) and constant $m$ in Eq. (15) are taking to be 1.

\subsection{The effect of heater size $\left(A_{\text {base }}\right)$, geometry and fluid-surface combination}

The impact of heater size on CHF when boiling methanol on a flat square copper surface has been experimentally investigated by Lienhard et al. (Lienhard, Dhir and Riherd, 1973). Their data alongside the correlation they suggested, as in Eq. 22 (Lienhard, Dhir and Riherd, 1973), are plotted in Fig. 8. 


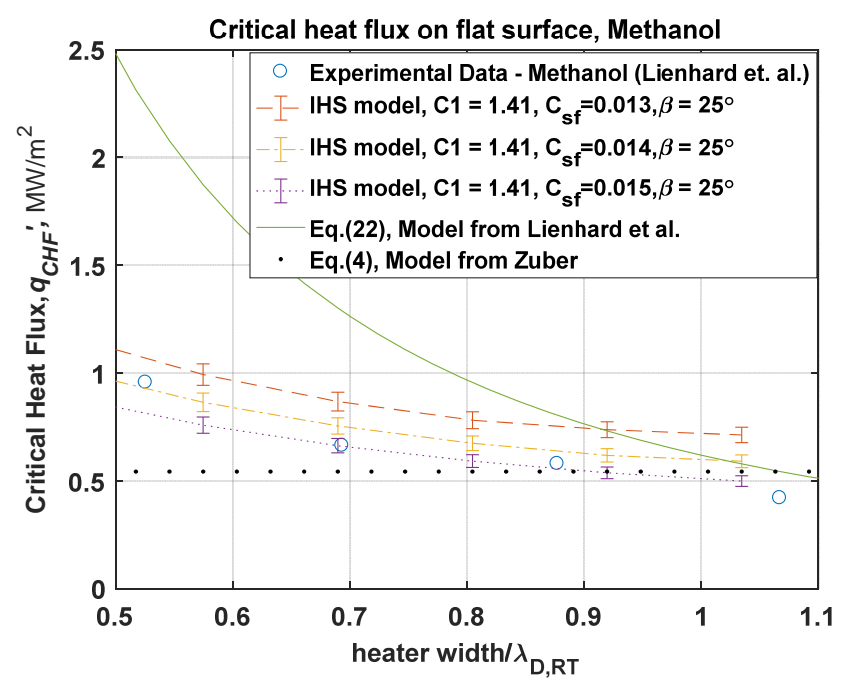

Fig. 8 The critical heat flux when boiling methanol on the flat square surface with different widths. Error bars represent 5\% uncertainty

$\frac{q_{C H F, L}^{\prime \prime}}{q_{C H F, Z}^{\prime \prime}}=1.14 \cdot \frac{\lambda_{R T, D}^{2}}{A_{\text {heater }}}$ when $\frac{L}{\lambda_{R T, D}}<\sqrt{2}$

where $A_{\text {heater }}$ is the heater area and $q_{C H F, Z}^{\prime \prime}$ is calculated by Eq. (4)

Fig. 8 also shows the $q_{C H F}^{\prime \prime}$ calculated by the IHS model assuming the contact angle is $25^{\circ}$, as reported in literature for methanol-copper combination (Shakir and Thome, 1986), and three different $C_{s f}$ value. No data on the exact $C_{s f}$ value for methanol-copper combination can be found in literature. Experiments data reported by Bailey et al. (Bailey et al., 2006) suggests the excessive temperature when boiling methanol is slightly higher than boiling water, so the $C_{s f}$ can be slightly higher than 0.013 , which is for the copper-water combination. Fig. 8 shows the proposed IHS model can better predict the CHF than Eq. (22). Fig. 8 also shows the predicted $q_{C H F}^{\prime \prime}$ reduces as $C_{s f}$ increases. This is because a bigger $C_{s f}$ will lead to a higher surface temperature at the same heat flux. This in turn will increase the bubble growth rate and therefore the instability 
wavelength, which eventually lead to a smaller critical vapour velocity and the required heat flux. The IHS model with $C_{S f}=0.014$ can predict most $q_{C H F}^{\prime \prime}$ within $5 \%$ uncertainty.

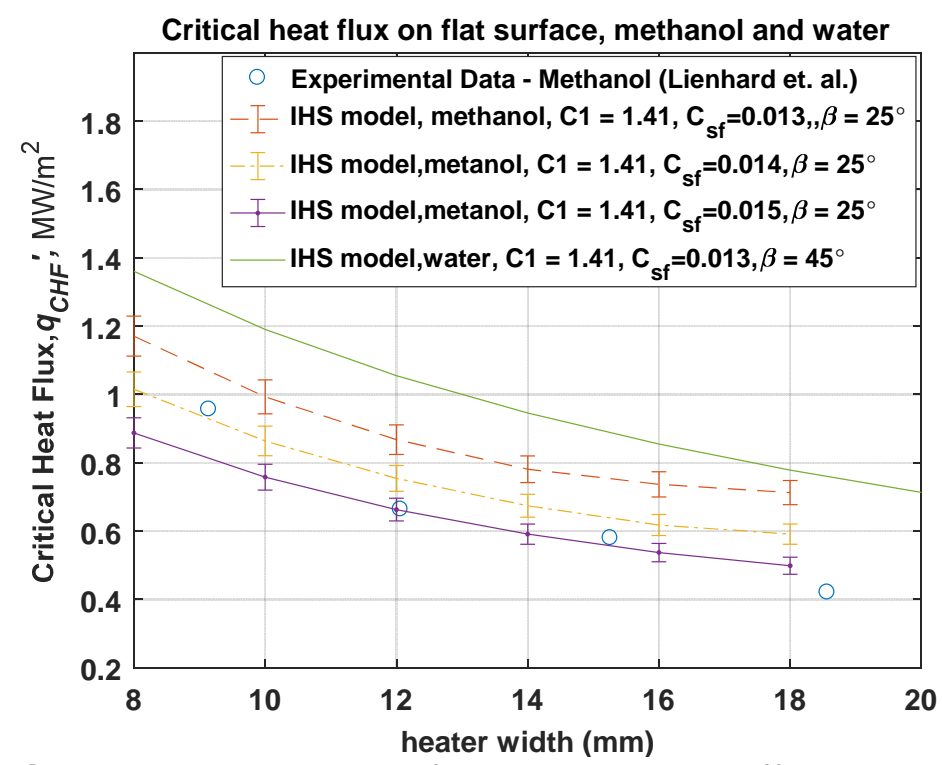

Fig. 9 The critical heat flux when using different liquids, liquid-surface combination on a square flat heating surface with different widths. Error bars represent $\pm 5 \%$ uncertainty

Fig. 9 shows the comparison of $q_{C H F}^{\prime \prime}$ on the same size heater when boiling water and methanol. It shows that the $q_{C H F}^{\prime \prime}$ when boiling water is much higher than boiling methanol primarily due to the larger evaporation enthalpy for water. Sun and Lienhard (Sun and Lienhard, 1970) also investigated the effect of heater size on the CHF when boiling methanol on a horizontal cylinder with different diameters. Their data and their empirical correlation, as in Eq. (23) (Sun and Lienhard, 1970), are plotted in Fig. 10.

$\frac{q_{C H F}^{\prime \prime}}{q_{C H F, Z}^{\prime \prime}}=0.89+2.27 \cdot \exp \left(-3.44 \cdot \sqrt{R^{\prime}}\right) ; R^{\prime}=\frac{R \cdot \sqrt{3} \cdot 2 \pi}{\lambda_{R T, D}}$ when $R^{\prime}>0.15$

where $R$ is the cylinder radius 


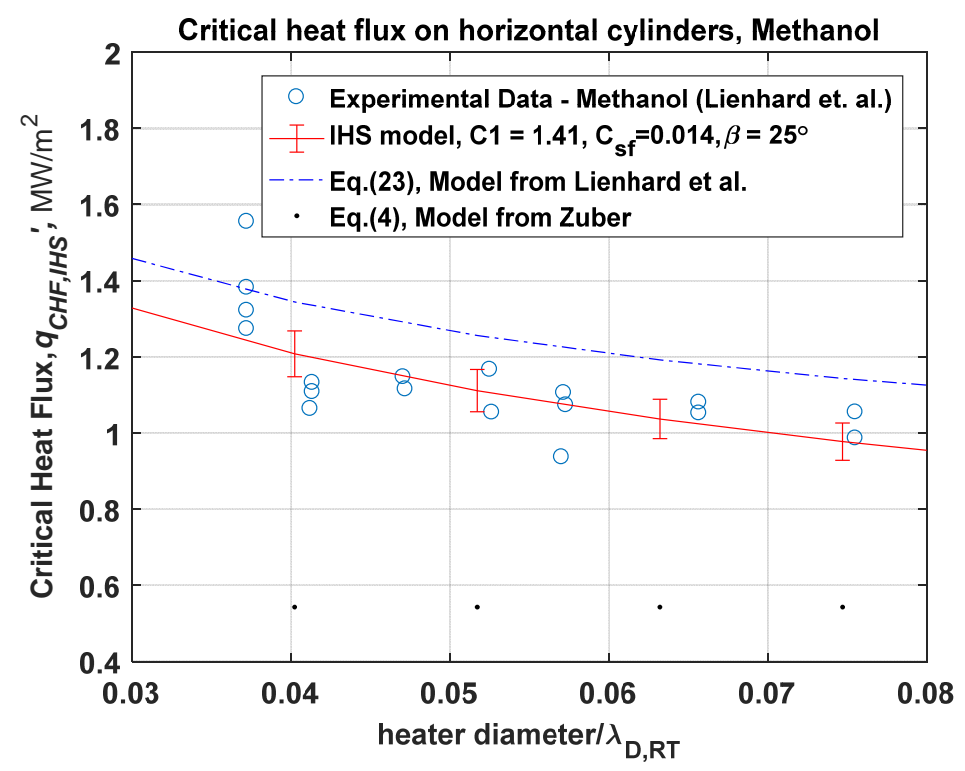

Fig. 10 The critical heat flux when boiling methanol on a long horizontal cylinder with different diameters. Error bars represent 5\% uncertainty

The results predicted by the IHS model $\left(C_{1}=1.41, C_{s f}=0.014, \beta=25^{\circ}\right)$ are also plotted in Fig. 10. For a long horizontal cylinder, the $A_{\text {base }}$ in the IHS model is calculated as:

$A_{\text {base }}=2 \pi R \cdot \lambda_{R T, D}$ when $D \leq \lambda_{R T, D} ;$ otherwise, $A_{\text {base }}=\lambda_{R T, D}^{2}$

Fig. 10 shows the IHS model can well predict the $q_{C H F}^{\prime \prime}$ with uncertainty smaller than the scattering of the data and the reported values are much larger than the ones calculated by Zuber's model.

In summary, the proposed IHS model can predict the $q_{C H F}^{\prime \prime}$ on both small flat surfaces and long horizontal cylinders within $5 \%$ uncertainty by using the actual heating surface area. The results also highlight the importance of knowing the actual heating surface area when extrapolating the $q_{C H F}^{\prime \prime}$ data from literature. In order to better predict the $q_{C H F}^{\prime \prime}$ using the IHS model, more accurate model and 
data are needed to predict the average surface temperature and contact angle on different liquid-surface combinations.

\subsection{The effect of contact angle}

The effect of contact angle on the CHF condition has been experimentally investigated by various researchers (Liaw and Dhir, 1986)(Hsu and Chen, 2012) and modelled by Kandlikar (Kandlikar, 2001) and Liao et al. (Liao, Bao and Liu, 2008). Kandlikar's model has been detailed in Eq. (5). Liao's model is an empirical model based on Zuber's model and is formulated in Eq. (25).

$$
q_{C H F, L}^{\prime \prime}=q_{C H F, Z}^{\prime \prime} \cdot\left(-0.73+\frac{1.73}{1+10^{-0.021(185.4-\theta)}}\right) \cdot\left(1+\frac{55-\beta}{100}(0.56-0.0013 \theta)\right)
$$

where $\theta$ is the surface inclination angle in degree ( 0 for the horizontal surface);

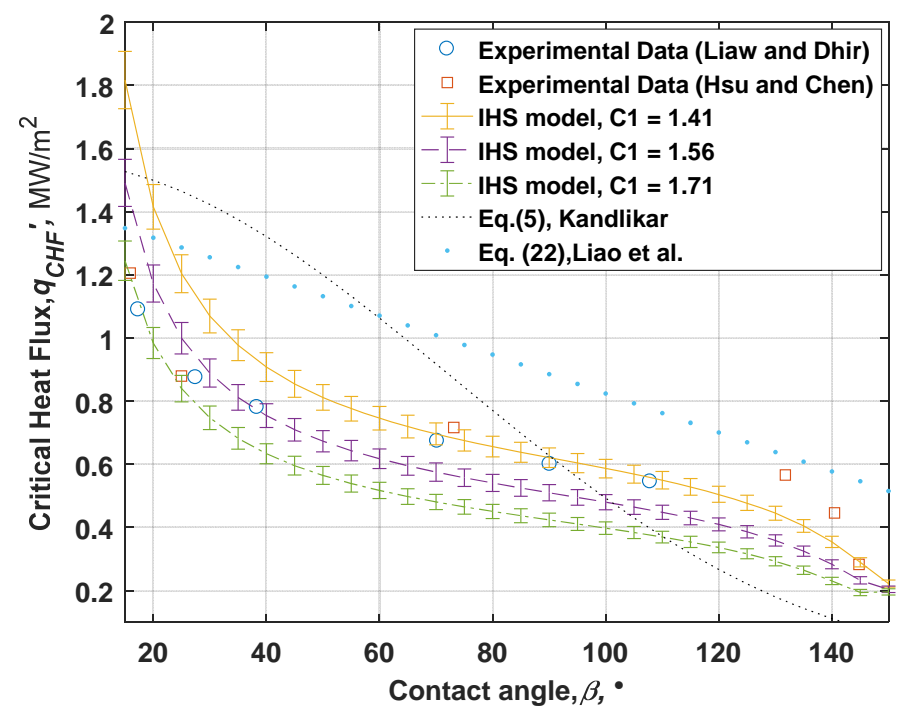

Fig. 11 The effect of contact angle on the critical heat flux. In the IHS model, $A_{\text {base }}=16 \times 16 \mathrm{~mm}^{2}, C_{s f}=0.013$. The $A_{\text {base }}$ is corresponding to the actual heater size. Error bars represent 5\% uncertainty

The current IHS model incorporates the effect of changing contact angle by tracing the detailed bubble merging process which causes the interface 
instability, as in Eq. (13) and (20). The comparison between Kandlikar's model, Liao's model and the current IHS model with different coefficient $C_{1}$ are shown in Fig. 11 against the experimental data reported by Liaw and Dhir (Liaw and Dhir, 1986) and Hsu and Chen (Hsu and Chen, 2012) when boiling water on copper surfaces pre-treated to get different wettability.

Fig. 11 shows the IHS model can predict most $q_{C H F}^{\prime \prime}$ for both hydrophobic and hydrophilic surface with $10 \%$ uncertainty, which is considerably better than the Kandlikar's model and the model developed by Liao et al. Fig. 11 also shows the critical heat flux increases as the contact angle reduces. This is because reducing the contact angle will increase the $S_{r e}$. In order to achieve CHF, the NB will need to grow faster to catch up with the detached LCB, which will require a higher heat flux. In addition, Fig. 11 shows a larger $C_{1}$ (i.e. higher bubble growth rate) leads to a lower CHF value because the required heat flux with higher bubble growth rate condition is smaller due to the increased instability wavelength. The IHS with $C_{1}=1.41$ (Ouwerkerk's model (van Ouwerkerk, 1971)) fits best to the data for contact angle larger than $60^{\circ}$, and IHS with $C_{1}=1.55$ (average value between Cooper's model (Cooper, 1969) and Ouwerkerk's model) fits best to the data for contact angle smaller than $60^{\circ}$. To better predict the $q_{C H F}^{\prime \prime}$ using the proposed IHS model, more accurate data and model to get the contact angle will need to be developed.

\subsection{The effect of system pressure}

The effect of system pressure on the critical heat flux has been investigated by various researchers (Sakurai and Fukuda, 2002; Bailey et al., 2006). Due to the limited amount of data available for the effect of pressure on bubble growth rate, 
it is difficult to find the correlation between the growth coefficient and the system pressure. Therefore, an empirical correlation, as in Eq. (26) has been developed through curve fittings to the data reported by Bailey et al. (Bailey et al., 2006), which are for pool boiling of water on a $1 \mathrm{~cm}^{2}$ square nickel coated copper disk up to 3 bar pressure. The contact angle was not reported by the author. Therefore, it is calculated as $35^{\circ}$ by assuming the $C_{1}=1.41$ in atmospheric pressure. Considering the rigorous cleaning process reported by the author and the high surface energy of the metal, the calculated contact angle seems plausibly higher than the typical contact angle on commercial water-copper surface $\left(45^{\circ}-55^{\circ}\right)$.

$C_{1}=1.21 \cdot P+0.19 ; P$ is the pressure on unit of Bar

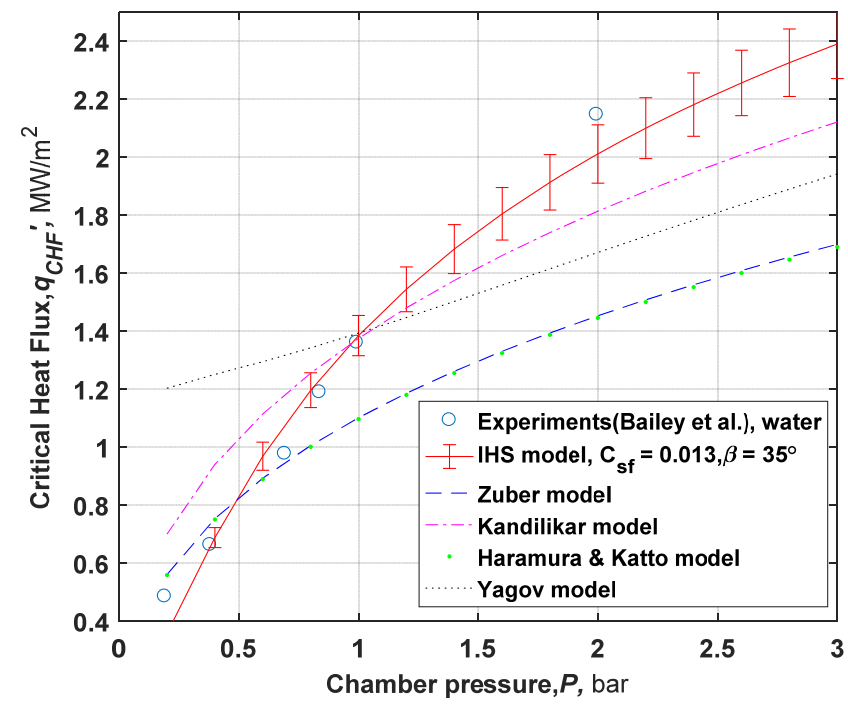

Fig. 12 The critical heat flux in different pressures when boiling water on a flat surface. Error bars represent 5\% uncertainty

The $q_{C H F}^{\prime \prime}$ calculated from the IHS model using Eq. (26) are shown in Fig. 12 together with the original data by Bailey et al. (Bailey et al., 2006) and different types of existing models. The plot shows that this simple linear correlation can 
be used to predict the $q_{C H F}^{\prime \prime}$ within 5\% uncertainty. It also shows all other types of models can not accurately capture the effect of changing pressure.

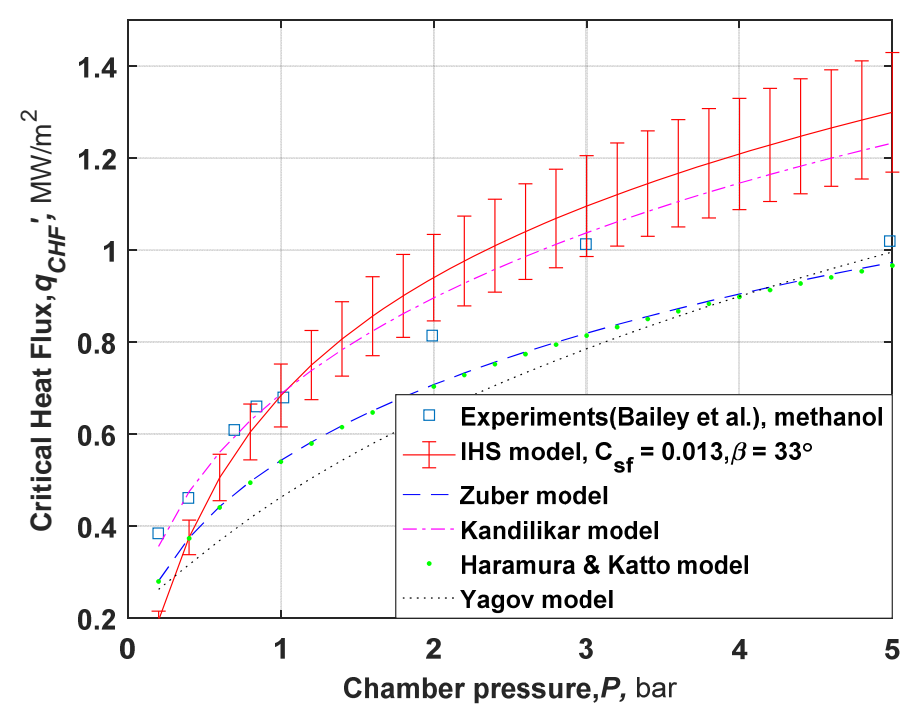

Fig. 13 The critical heat flux in different pressures when boiling methanol on a flat surface. Error bars represent $10 \%$ uncertainty

To further validate the results, the same IHS model using $C_{1}$ calculated from Eq.

(26) has been applied to predict the $q_{C H F}^{\prime \prime}$ on the same heating surface when boiling methanol up to 5 bar pressure. The results are shown in Fig. 13. It shows the IHS model can generally predicted the $q_{C H F}^{\prime \prime}$ within $10 \%$ uncertainty. The Kandlikar model with $\beta=33^{\circ}$ fits the data very well, which indicates the hydrodynamic model incorporating the effect of contact angle has the potential to capture the effect of pressure within a certain range.

To understand the effect of pressure on IHS model, the growth rate coefficients (i.e. $\gamma$ in Eq. (14)) for both water and methanol are plotted in Fig. 14. It shows the bubble growth rates will increase significantly in low pressure, which can lead to a bigger instability wavelength so the required vapour jet velocity and the heat flux is reduced significantly. 


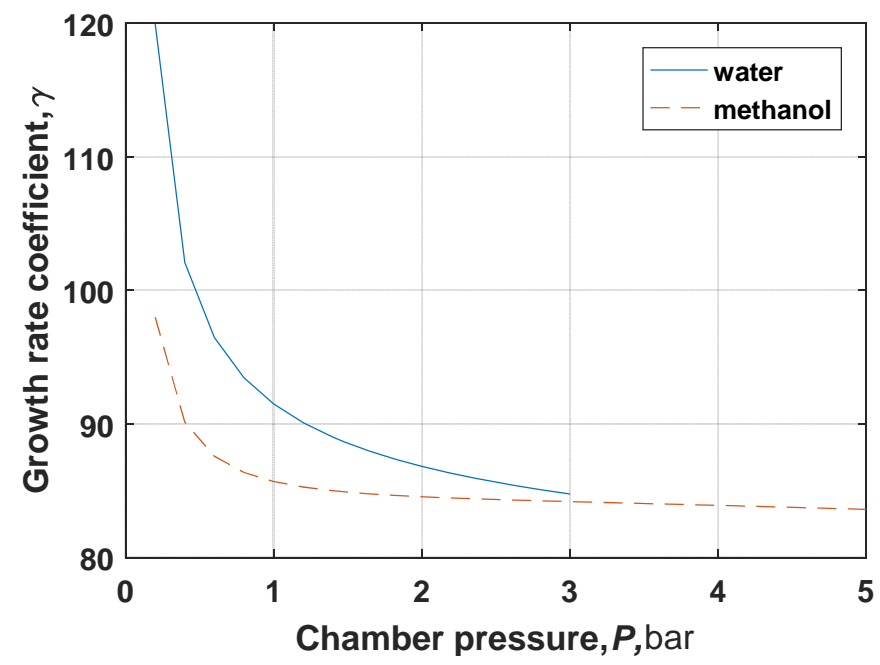

Fig. 14 The growth rate coefficient in different pressure

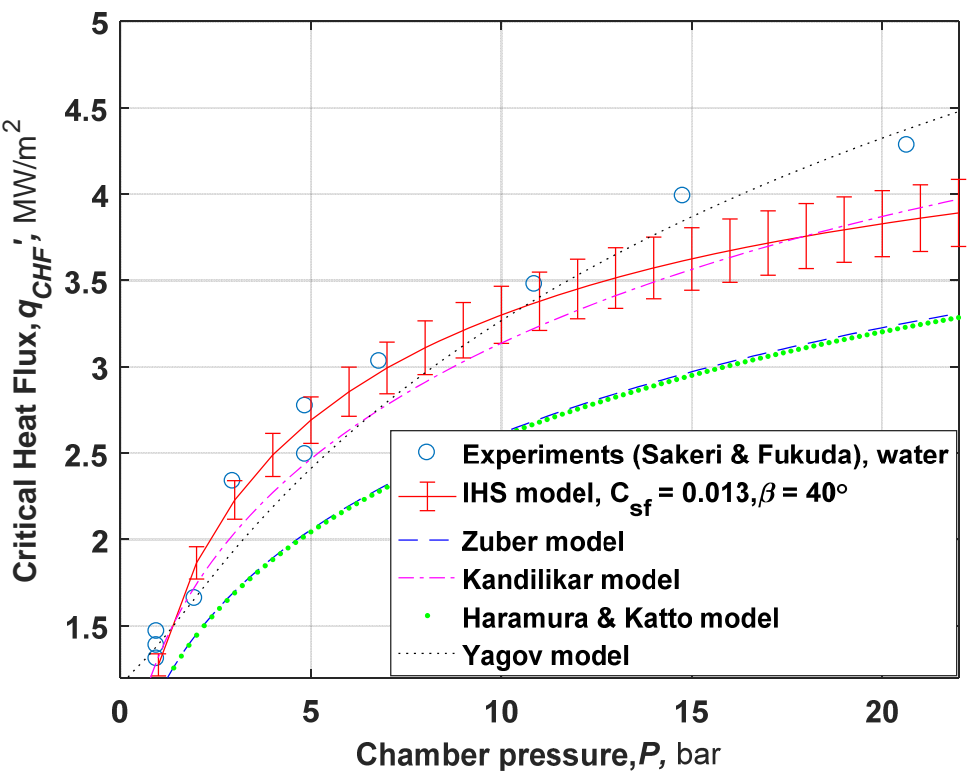

Fig. 15 The critical heat flux in different pressures when boiling methanol on a flat surface, error bars represent $5 \%$ uncertainty

The IHS model with Eq. (26) have also been applied to predict the $q_{C H F}^{\prime \prime}$ when boiling water on small horizontal cylinders in different pressures. The results, together with the experimental data reported by Sakurai and Fukuda (Sakurai and Fukuda, 2002) (water boiling on a $1.2 \mathrm{~mm}$ diameter titanium cylinder), are shown in Fig. 15. It shows the IHS model can predict the $q_{C H F}^{\prime \prime}$ within $5 \%$ 
uncertainty up to 10 bar and the error starts to increase in higher pressure. This can be due to the assumption behind Eq. (16) where the inertial force and buoyancy force are much larger than other forces. This assumption will be less valid in very high pressure because the inertial force will become much smaller due to the slow growth rate. As a result, the drag force will need to be taken into account in the bubble growth model.

\subsection{Validation of different semi-empirical models}

The model proposed in this paper includes many semi-empirical correlations to predict the detailed bubble behaviours and the surface temperature.

Considering the incredibly complex two-phase mixing and heat transfer process in CHF conditions, detailed modelling which does not rely on any empirical coefficients is a formidable task if possible. As the first step to test the validity of the assumptions that CHF is triggered by the hydrodynamic instability induced IHS, these semi-empirical correlations have been carefully selected to investigate the effect of different surface characteristics (e.g. wettability, size and geometry) on CHF. Inevitably, these correlations can potentially lead to significant errors outside the original experimental conditions where the coefficients are obtained. In this section, the detailed parameters, including the dry area, the instability breakup length and the surface temperatures, predicted by the IHS models will be compared to the experimental data reported in the literature. The results shown in this section use Eq. (26) to calculate $C_{1}$, the constant $C$ in Eq. (12) and constant $m$ in Eq. (15) are both taking to be 1. 
Although a significant amount of experimental work has been reported in the literature, a single experimental work including all the required information (e.g. contact angle, heater size and geometry, detailed bubble behaviours and the surface temperature) cannot be found. The only detailed experimental results which include the majority of the parameters was reported by Chu et al. (Chu et al., 2014). It includes all other information except for the surface temperature.

The comparison between the experimental results (including the data extracted from Fig. 4 (b)) and the results calculated by the IHS model is shown in Table 2. The IHS model takes the measured contact angle, the heater size and geometry as the inputs and calculates the CHF and detailed bubble behaviours. $C_{s f}$ is the only input parameter that cannot be determined directly from the reported data, i.e. there is no $C_{s f}$ value reported for water-ITO coating. In table 2 , the value of $C_{s f}$ is chosen to fit for the CHF in test 2 condition and then remain unchanged for test 3 calculation. Table 2 shows that the chosen semiempirical correlations can well predict the related bubble size (i.e. Eq. (14), (19) and (20)) regardless of greatly simplified assumptions. However, the predicted instability wavelength (i.e. $h_{\text {neck }}$ ) is smaller than the measured value which can be due to the inaccuracy of the assumption that NB occurs at $1 \mathrm{~mm}$ thick superheated layer. More experimental data will need to further validate this assumption. In comparison, the instability wavelength used in all other existing hydrodynamic based models is $27.3 \mathrm{~mm}$, which is more than 4 times bigger than the observed experimental results and can lead to significant error in predicting the required vapour velocity. 
Table 2 Comparison of the IHS modelling results and the experimental data reported in literature

\begin{tabular}{|l|l|l|l|l|l|}
\hline & $\begin{array}{l}\text { CHF } \\
\left(\mathrm{W} / \mathrm{cm}^{2}\right)\end{array}$ & $\begin{array}{l}A_{\text {neck }} \\
\left(\mathrm{mm}^{2}\right)\end{array}$ & $\begin{array}{l}h_{-} \text {neck } \\
(\mathrm{mm})\end{array}$ & $\begin{array}{l}R_{L C B} \text {-base } \\
(\mathrm{mm})\end{array}$ & $\tau_{g}(\mathrm{~ms})$ \\
\hline $\begin{array}{l}\text { Chu et al. (Chu et al., } \\
\text { 2014), test 2, } \beta=68^{\circ}\end{array}$ & 826 & $\sim 12.4$ & $\sim 6.2$ & $4.5-5.5$ & $14-20$ \\
\hline $\begin{array}{l}\text { IHS model for test 2, } \\
C_{s f}=0.0123\end{array}$ & 829 & 12.2 & 5.1 & 5.4 & 15.4 \\
\hline $\begin{array}{l}\text { Chu et al. (Chu et al., } \\
\text { 2014), test 3, } \beta=53^{\circ}\end{array}$ & 933 & $\begin{array}{l}\text { not } \\
\text { available }\end{array}$ & $\begin{array}{l}\text { not } \\
\text { available }\end{array}$ & $4.5-6.5$ & $\begin{array}{l}\text { Not } \\
\text { available }\end{array}$ \\
\hline $\begin{array}{l}\text { IHS model for test 3, } \\
C_{s f}=0.0123\end{array}$ & 928 & 15.0 & 6.2 & 5.4 & 15.2 \\
\hline
\end{tabular}

Although the comparison in table 2 gives more confidence on the bubble growth and hydrodynamic instability models, it does not provide any validation on the Rohsenow correlation and the related empirical coefficients (i.e. $C_{s f}$ and $m$ ) because these coefficients are determined by fitting the CHF value in test 2 . In fact, there is an ongoing debt on whether the Rohsenow correlations can predict the heat flux at different excess temperature accurately. Pioro et al. compared the accuracy of different pool correlations over a wide range of reported experimental conditions (Pioro, Rohsenow and Doerffer, 2004). They concluded that the Rohsenow correlation with well-documented coefficients is still the most accurate correlation and its RMS error in predicting the heat flux with known surface temperature when boiling water on different types of surfaces is less than $20 \%$. Considering that the IHS model is use the Rohsenow correlation to predict the excess surface temperature $\left(\Delta T_{s}\right)$ with known heat flux $(q)$ and $\Delta T_{s} \propto$ $q^{0.33}$, the error in predicting the surface temperature should be less than $7 \%$. However, when the Rohsenow correlations are used in boiling conditions 
different from the original experimental conditions where $C_{s f}$ and $m$ are determined, the accuracy can be significantly deteriorated so more work will need to be done to find the $C_{s f}$ and $m$ in different boiling conditions or to develop more accurate boiling models which are valid for wider ranges of boiling conditions.

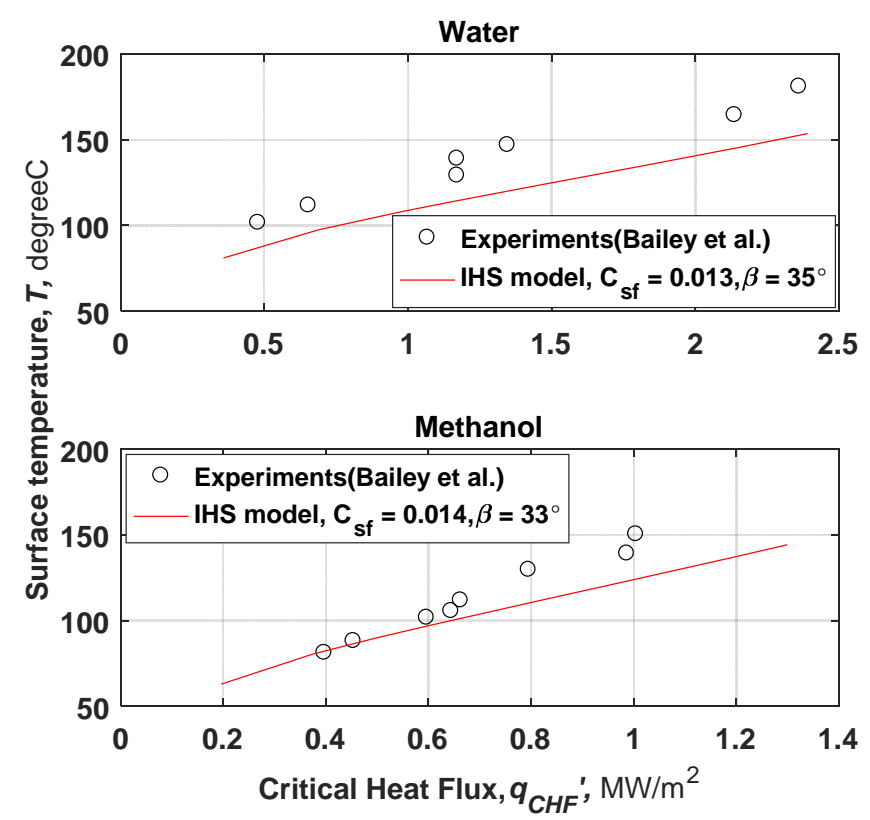

Fig. 16 The surface temperature - critical heat flux correlation when boiling water and methanol on a flat surface in difference pressures

The experimental work reported by Bailey et al. (Bailey et al., 2006) includes data on both surface temperature and heat flux. The comparison between their experimental data and the results predicted by the IHS model is shown in Fig. 16. It shows that the surface temperature predicted by the Rohsenow correlations with specified $C_{s f}$ and $m$ are generally lower than the measured value. Such a comparison is not strictly rigorous because the measured temperature is a time-averaged value from a single point while the temperature predicted by the IHS model is the typical surface temperature underneath the 
superheated liquid microlayer which contribute to the bubble growth. Therefore, the time-averaged temperature (with both dry and wet conditions) could be understandably higher than the average temperature which affects the bubble growth. A more rigorous comparison should be made between the timeaveraged wet temperature and the temperature predicted by the IHS model. However, we could not find such a data in literature with detailed surface characteristics.

\section{Conclusions}

A new model based on the hydrodynamic instability induced irreversible hot spot (IHS) model has been presented in this paper. Based on the experimental observations that whether the CHF condition occurs is depending on whether there is any growing IHS, this model suggests the IHS is triggered by consistent bubble separation process triggered by the consistent hydrodynamic instability.

The key findings and implications of this new model are as follows:

1. The instability wavelengths in CHF conditions depend on the bubble growth and departure process. This feature has been observed experimentally and reported by many researchers. Therefore, the assumption on the fixed instability wavelength behind the existing hydrodynamic instability models is not accurate.

2. The fundamental equation (i.e. Eq. (12)) of the IHS model fits the reported experimental data well.

3. The effect of heater size on the critical heat flux can be significant when the characteristic dimension of the heater is smaller than the most 
dangerous wavelength predicted by the Rayleigh-Taylor instability (i.e. $\left.\lambda_{R T, D}\right)$. The IHS model can predict most $q_{C H F}^{\prime \prime}$ value on small flat surface and long horizontal cylinders when boiling methanol to within 5\% uncertainty. The model suggests the higher $q_{C H F}^{\prime \prime}$ observed on smaller size heating surfaces is caused by the combined effect of increased bubble growth rate and availability of heating area.

4. The effect of contact angles on the critical heat flux on a flat surface can be predicted by the IHS models to within 5-10\% uncertainty for both the hydrophilic and hydrophobic surfaces. As the contact angle reduces, the required bubble growth rate needs to be higher to induce the hydrostatic instability, which requires a higher heat flux.

5. A linear empirical correlation (Eq.(15)) for the bubble growth rate constant $\left(C_{1}\right)$ has been developed through curve fitting to existing data for the boiling of water. The IHS model incorporating this correlation can predict the $q_{C H F}^{\prime \prime}$ when boiling of water on flat surfaces and horizontal cylinders to within $5 \%$ uncertainty for pressures up to 10 bar. It can predict the $q_{C H F}^{\prime \prime}$ when boiling methanol on flat surface within $10 \%$ uncertainty. As the system pressure increases, the bubble growth rate is reduced so a higher heat flux is required to initiate the hydrodynamic instability.

6. The detailed parameters (e.g. bubble size, neck size, neck height, growth time) predicted by IHS model are in good agreement with the limited amount of data reported in the literature. The comparison suggests the following values of the coefficients to be used in the IHS model: $n=$ 
$0.5, C=1$. The $C_{s f}$ and $m$ value in Eq. (15) have been well-documented in literature (Pioro, 1998; Carey, 2008). More detailed experimental data are required to further validate the sub-models.

In summary, the proposed IHS model can capture the effects of heating surface area, contact angle and system pressure on the critical heat flux by tracing the change of the dominant physical processes. As more accurate and detailed experimental data and correlations become available to predict the bubble growth rates in different boiling conditions, this model can be further improved.

\section{Acknowledgement}

We would like to thank our colleagues, Mr Hendrik Versteeg and Prof. Colin Garner, for their kind help in technical discussion and manuscript preparation. 


\section{Appendix 1: Derivation of Eq. (21) for constant heat flux condition}

The required liquid superheat for a growing bubble nuclei, as shown in Fig. A1, is solved by Davis and Anderson (Davis and Anderson, 1966) according to the Clausius - Clapeyron equation and the Young's equation:

$T_{c}=T_{s a t}+\frac{R T_{C} T_{s a t}}{h_{l v}} \ln \left(1+\frac{2 \sigma(1+\cos \beta)}{P \cdot y^{\prime}}\right)$

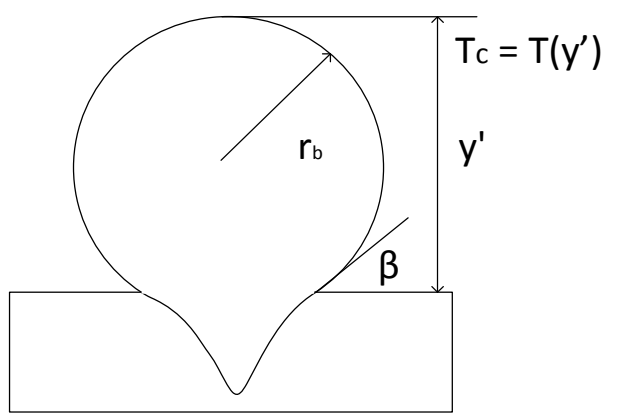

Fig. A1 Superheat requirement for a growing bubble nuclei (Davis and Anderson, 1966)

Assuming a linear temperature distribution for $y \leq y^{\prime}$ and the temperature gradient required by the liquid superheat condition equals to the temperature profile at the top of the nuclei, the wall temperature $\left(T_{w, c}\right)$ can be calculated as:

$$
\begin{aligned}
& T_{w, c}=T_{c}+q_{w}^{\prime \prime} \cdot \frac{y^{\prime}}{k_{l}}=T_{s a t}+\frac{R T_{s a t}^{2}}{h_{l v}} \ln (1+\varepsilon) /\left[1-\frac{R T_{s a t}}{h_{l v}} \ln (1+\varepsilon)\right]+q_{w}^{\prime \prime} \cdot \frac{y^{\prime}}{k_{l}} \\
& \varepsilon=\frac{2 \sigma \cdot(1+\cos (\beta))}{P \cdot y^{\prime}} ; y^{\prime}=\frac{1+\cos (\beta)}{P}+\left[\left(\frac{(1+\cos (\beta)) \sigma}{P}\right)^{2}+\frac{2(1+\cos (\beta)) k_{l} \sigma T_{s a t}}{q_{w}^{\prime \prime} h_{l v} \rho_{v}}\right]^{0.5}
\end{aligned}
$$

This required liquid superheat condition is achieved by heating up the saturated liquid during the waiting time $\left(\tau_{w}\right)$ through transient heat conduction. Assuming the heat transfer process is from the wall to the semi-infinite region under 
constant heat flux condition, the wall superheat can be calculated by the wellknown solution:

$T_{w}(t)=T_{i}+\frac{2 q_{w}}{k} \sqrt{\frac{\alpha t}{\pi}}$

where $T_{i}$ is the initial temperature

If $T_{i}=T_{f}$ and $T_{w}\left(\tau_{w}\right)=T_{w, c}$, then

$\tau_{w}=\left[\left(T_{w, c}-T_{f}\right) \cdot \frac{k_{l}}{2 \cdot q_{w}^{\prime \prime}}\right]^{2} \cdot\left(\frac{\pi}{\alpha_{l}}\right) ;$ 


\section{References:}

Ahn, H. S. and Kim, M. H. (2012) 'Visualization study of critical heat flux mechanism on a small and horizontal copper heater', Int. J Multiphase Flow, 41, pp. 1-12.

Bailey, W. et al. (2006) 'Pool boiling study on candidature of pentane, methanol and water for near room temperature cooling', Thermal and Thermomechanical Proceedings 10th Intersociety Conference on Phenomena in Electronics Systems, 2006. ITHERM 2006. IEEE, pp. 599603.

Brusstar, Matthew J.; Merte, H. (1994) 'Effects of buoyancy on the critical heat flux in forced convection', J. thermophys heat tr, 8(2).

Carey, V. P. (2008) Liquid-vapor phase change phenomena.

Choi, J. Y., No, H. C. and Kim, J. (2016) 'Development of a dry patch model for critical heat flux prediction', Int. J. Heat Mass Tran. 100, pp. 386-395.

Chu, I. C. et al. (2014) 'Observation of critical heat flux mechanism in horizontal pool boiling of saturated water', Nucl. Eng. Des. 279, pp. 189-199.

Chu, I. C., No, H. C. and Song, C. H. (2013) 'Visualization of boiling structure and critical heat flux phenomenon for a narrow heating surface in a horizontal pool of saturated water', Int. J. Heat Mass Tran. 62(1), pp. 142152.

Cooper, M. G. (1969) 'The microlayer and bubble growth in nucleate pool boiling', Int. J. Heat Mass Tran., 12(8), pp. 915-933.

Cooper, M. G. and Lloyd, A. J. P. (1969) 'The microlayer in nucleate pool boiling', Int. J. Heat Mass Tran., 12(8), pp. 895-913.

Davidson, J. F. and Schuler, B. O. G. (1960) 'Bubble formation at an orifice in a viscous liquid', Trans. Instn. Chem. Engrs., 38(9), pp. 1748-1750.

Davis, E. J. and Anderson, G. H. (1966) 'The incipience of nucleate boiling in forced convection flow', AIChE Journal, 12(4), pp. 774-780.

Dhillon, N. S., Buongiorno, J. and Varanasi, K. K. (2015) 'Critical heat flux maxima during boiling crisis on textured surfaces', Nature Communications. 6, p. 8247.

Dhir, K. and Lienhard, H. (1973) Extended hydrodynamic theory of the peak and minimum pool boiling heat fluxes. NASA Contractor Report, NASA CR2270.

El-Genk, Mohamed S.; Guo, Z. (1993) 'Transient boiling from inclined and downward-facing surfaces in a saturated pool', Int. J. Refrig., 16(6).

Fang, X. and Dong, A. (2016) 'A comparative study of correlations of critical heat flux of pool boiling', Journal of Nuclear Science and Technology, 3131(July), pp. 1-12.

Haramura, Y. and Katto, Y. (1983) 'A new hydrodynamic model of critical heat flux, applicable widely to both pool and forced convection boiling on submerged bodies in saturated liquids', Int. J. Heat Mass Tran., 26(3), pp. 389-399.

Hsu, C.-C. and Chen, P.-H. (2012) 'Surface Wettability Effects on Critical Heat Flux of Boiling Heat Transfer using Nanoparticle Coatings', Int. J. Heat Mass Tran., 55(13-14), pp. 3713-3719. 
Kandlikar, S. G. (2001) 'A Theoretical Model to Predict Pool Boiling CHF Incorporating Effects of Contact Angle and Orientation', J. Heat Transf., 123(6), p. 1071.

Kim, D. E., Song, J. and Kim, H. (2016) 'Simultaneous observation of dynamics and thermal evolution of irreversible dry spot at critical heat flux in pool boiling', Int. J. Heat Mass Tran., 99, pp. 409-423.

Kutateladze, S. S. (1950) 'Hydromechanical model of heat transfer crisis in pool liquid boiling', J. Tech. Phys., 20(11).

Liang, G. and Mudawar, I. (2017) 'Pool boiling critical heat flux (CHF) - Part 1: Review of mechanisms, models, and correlations', Int. J. Heat Mass Tran. Elsevier Ltd.

Liao, L., Bao, R. and Liu, Z. (2008) 'Compositive effects of orientation and contact angle on critical heat flux in pool boiling of water', Heat and Mass Transfer/Waerme- und Stoffuebertragung, 44(12), pp. 1447-1453.

Liaw, S. P. and Dhir, V. K. (1986) 'Effect of surface wettability on transition boiling heat transfer froma vertical surface', Proceedings of the 8th International Heat and Mass Transfer, 4, pp. 2031-2036.

Lienhard, H., Dhir, V. K. and Riherd, D. (1973) 'Peak Pool Boiling Heat -Flux Measurements on Finite Horizontal Flat Plates', J. Heat Transf., (November), pp. 477-482.

Mikic, B. B., Rohsenow, W. M. and Griffith, P. (1970) 'On bubble growth rates', Int. J. Heat Mass Tran., pp. 657-666.

van Ouwerkerk, H. J. (1971) 'The rapid growth of a vapour bubble at a liquidsolid interface', Int. J. Heat Mass Tran., 14(9), pp. 1415-1431.

Pioro, I. L. (1998) 'Experimental evaluation of constants for the Rohsenow pool boiling correlation', Int. J. Heat Mass Tran., 42(11), pp. 2003-2013.

Pioro, I. L., Rohsenow, W. and Doerffer, S. S. (2004) 'Nucleate pool-boiling heat transfer. I: review of parametric effects of boiling surface', Int. J. Heat Mass Tran., 47(23), pp. 5033-5044.

Plesset, M. S. and Zwick, S. A. (1954) 'The growth of vapor bubbles in superheated liquids', J. Appl. Phys., 25(4), pp. 493-500.

Rohsenow, W. M. (1951) 'A method of correlating heat transfer data for surface boiling of liquids', J. Heat Transf., 74, pp. 969-976.

Sakashita, $H$. and Ono, A. (2009) 'Boiling behaviors and critical heat flux on a horizontal plate in saturated pool boiling of water at high pressures', Int. J. Heat Mass Tran., 52(3-4), pp. 744-750.

Sakurai, A. and Fukuda, K. (2002) 'Mechanisms of Subcooled Pool Boiling CHFS Depending on Subcooling, Pressure, and Test Heater Configurations and Surface Conditions in Liquids', Heat Transfer, Volume 3, 2002, pp. 213-225.

Shakir, S. and Thome, J. R. (1986) 'Boiling nucleation of mixtures on smooth and enhanced surfaces', in Proceedings of the eighth international heat transfer conference. San Francisco.

van Stralen, S. J. D. et al. (1975) 'Bubble growth rates in pure and binary systems: Combined effect of relaxation and evaporation microlayers', Int. J. Heat Mass Tran., 18(3), pp. 453-467.

Sun, K. H. and Lienhard, J. H. (1970) 'The peak pool boiling heat flux on 
horizontal cylinders', Int. J. Heat Mass Tran., 13(9).

Theofanous, T. G. et al. (2002) 'The boiling crisis phenomenon part II: Dryout dynamics and burnout', Experimental Thermal and Fluid Science, 26(6-7), pp. 793-810.

Unal, C., Daw, V. and Nelson, R. a. (1992) 'Unifying the Controlling Mechanisms for the Critical Heat Flux and Quenching: The Ability of Liquid to Contact the Hot Surface', J. Heat Transf., 114(4), p. 972.

Wiebe, J. R. and Judd, R. L. (1971) 'Superheat Layer Thickness Measurements in Saturated and Subcooled Nucleate Boiling', J. Heat Transf., 93(4), p. 455.

Yagov, V. V. (1988) 'A physical model and calculation formula for critical heat fluxes with nucleate pool boiling of liquids', Therm. Eng., 35.

Yagov, V. V. (2014) 'Is a crisis in pool boiling actually a hydrodynamic phenomenon?', Int. J. Heat Mass Tran. Elsevier Ltd, 73, pp. 265-273.

Zijl, W. (1978) Departure of a bubble growing on a horizontal wall. Technische Hogeschool Eindhoven.

Zuber, N. (1959) 'HYDRODYNAMIC ASPECTS OF BOILING HEAT TRANSFER (thesis)', United States Atomic Energy Commision.

Zuber, N. (1961) 'The dynamics of vapor bubbles in nonuniform temperature fields', Int. J. Heat Mass Tran., 2(1-2), pp. 83-98.

\section{Figure captions}

Fig. 1 Force-imbalance on bubbles in CHF in Kandlikar's model

Fig. 2 Vapour structure near heated surface at high heat flux proposed by Haramura and Katto

Fig. 3 Schematic of the liquid meniscus at the interface in Yagov's model

Fig. 4 Bubble interface in CHF conditions. (a) Left-hand images show the side view of bubbles (white area), and the right-hand images show the distribution of the dry areas (black area) in heating surface. (b) The top image shows the side view of the bubble (white area), and the bottom images show the distribution of the dry areas (black area) in heating surface. Images are obtained by post-processing (contrast enhancing and binary image conversion) the photos reported in literature

Fig. 5 The two-dimensional periodic behaviour of bubbles in high heat flux nucleate boiling conditions. The bold dashed line indicates the dry area. The small box in stage (f) highlights the liquid-vapour interface where the large bubble merges with the growing nuclei bubbles 
Fig. 6 Hydrodynamic stability during the bubble merging process, i.e. stage (f) in Fig.5

Fig. 7 Cross-sectional view of the threshold condition for the bubble merging (spatial and temporal averaged behaviour)

Fig. 8 The critical heat flux when boiling methanol on the flat square surface with different widths. Error bars represent $5 \%$ uncertainty

Fig. 9 The critical heat flux when using different liquids, liquid-surface combination on a square flat heating surface with different widths. Error bars represent $\pm 5 \%$ uncertainty

Fig. 10 The critical heat flux when boiling methanol on a long horizontal cylinder with different diameters. Error bars represent $5 \%$ uncertainty

Fig. 11 The effect of contact angle on the critical heat flux. In the IHS model, $A_{\text {base }}=16 \times 16 \mathrm{~mm}^{2}, C_{s f}=0.013$. The $A_{\text {base }}$ is corresponding to the actual heater size. Error bars represent $5 \%$ uncertainty

Fig. 12 The critical heat flux in different pressures when boiling water on a flat surface. Error bars represent 5\% uncertainty

Fig. 13 The critical heat flux in different pressures when boiling methanol on a flat surface. Error bars represent $10 \%$ uncertainty

Fig. 14 The growth rate coefficient in different pressure

Fig. 15 The critical heat flux in different pressures when boiling methanol on a flat surface, error bars represent $5 \%$ uncertainty

Fig. 16 The surface temperature - critical heat flux correlation when boiling water and methanol on a flat surface in difference pressures

\section{List of Tables}

Table 1 Comparison between experimental data and Eq. (12)

Table 2 Comparison of the IHS modelling results and the experimental data reported in literature 
